\title{
The QCD sign problem and dynamical simulations of random matrices
}

\author{
Jacques Bloch and Tilo Wettig \\ Institute for Theoretical Physics, University of Regensburg, 93040 Regensburg, Germany \\ E-mail: jacques.bloch@physik.uni-regensburg.de, \\ tilo.wettig@physik.uni-regensburg.de
}

\begin{abstract}
At nonzero quark chemical potential dynamical lattice simulations of QCD are hindered by the sign problem caused by the complex fermion determinant. The severity of the sign problem can be assessed by the average phase of the fermion determinant. In an earlier paper we derived a formula for the microscopic limit of the average phase for general topology using chiral random matrix theory. In the current paper we present an alternative derivation of the same quantity, leading to a simpler expression which is also calculable for finite-sized matrices, away from the microscopic limit. We explicitly prove the equivalence of the old and new results in the microscopic limit. The results for finitesized matrices illustrate the convergence towards the microscopic limit. We compare the analytical results with dynamical random matrix simulations, where various reweighting methods are used to circumvent the sign problem. We discuss the pros and cons of these reweighting methods.
\end{abstract}

KEYworDs: Random matrix theory, Lattice QCD, Quark chemical potential 


\section{Contents}

1 Introduction 1

2 Random matrix model 2

3 Average phase of the fermion determinant 4

4 Solving the complex Cauchy transform $\quad 6$

$\begin{array}{llr}5 & \text { Microscopic limit } & 9\end{array}$

$6 \quad$ Equivalence of integration results 12

7 Dynamical random matrix simulations with complex weights $\quad 15$

$\begin{array}{lll}7.1 & \text { Computing averages using reweighting } & 15\end{array}$

$\begin{array}{lll}7.2 & \text { Numerical results } & 19\end{array}$

$\begin{array}{llr}8 & \text { Conclusions } & 20\end{array}$

$\begin{array}{ll}\text { A Relation between orthogonal polynomials } & 21\end{array}$

B Chiral limit $\quad 22$

$\begin{array}{lll}\text { B.1 Finite } N & 22\end{array}$

B.2 Microscopic limit 23

C Proof of integral relation (6.4) 24

$\begin{array}{ll}\text { D Proof of identity (6.8) } & 25\end{array}$

$\begin{array}{llr}\text { E Recurrence relation for } \mathcal{J}_{\nu, k} & 26\end{array}$

$\begin{array}{lr}\text { F Proof of identity (6.17) } & 29\end{array}$

G Error estimation for the random matrix simulations $\quad 31$

\section{Introduction}

In dynamical lattice simulations of QCD at nonzero quark chemical potential $\mu$ the generation of a Markov chain through importance sampling is hindered by the sign problem caused by the complex fermion determinant, see [1] for a review. The severity of the sign problem grows as $\mu$ increases and the determinant fluctuates more strongly. In the $\varepsilon$-regime 
of QCD, i.e., to leading order in the $\varepsilon$-expansion of chiral perturbation theory [2], the spectral properties of the Dirac operator are universal and can be computed in the microscopic limit of chiral random matrix theory (chRMT) [3-5]. This equivalence also holds at $\mu \neq 0$ so that chRMT can be used as a tool to investigate the sign problem.

The fluctuating behavior of the fermion determinant can be characterized by is its average phase. Using chRMT, Splittorff and Verbaarschot have computed the average phase at $\mu \neq 0$ for trivial topology in the quenched and unquenched case [6]. Their results were later extended to nonzero temperature [7] and to general topology [8]. The complex analysis employed in ref. [8] is quite involved, and in the present work we give an alternative derivation of the formula for general topology, based on ideas presented in ref. [9]. Although the final integral expressions for the microscopic limit of the average phase look quite different in both cases, we show that they are indeed equivalent. En-passant we also derive some interesting new integral identities. In addition, the new derivation also provides an analytical expression for the average phase of finite-sized matrices, away from the microscopic limit. This allows us to verify the analytical formulas numerically using dynamical chRMT simulations. Such simulations are very costly and can only be performed with high statistical accuracy for small-sized matrices. In our dynamical chRMT simulations the complex weights are implemented using various reweighting methods. We provide a discussion of the pros and cons of these methods.

The structure of this paper is as follows. In section 2 we introduce the chiral random matrix model with a chemical potential. In section 3 we show how the average phase of the fermion determinant can be computed in this model using complex Cauchy transforms. In section 4 the complex Cauchy transform is solved for finite-sized matrices, and in section 5 the microscopic limit is taken. In section 6 we prove the equivalence of the integral representations of the microscopic limits derived here and in ref. [8]. In section 7 we verify the analytical predictions for the unquenched case by random matrix simulations away from the microscopic limit, using different reweighting methods. Finally we draw conclusions in section 8. Intermediate steps of the calculations are worked out in several appendices.

\section{Random matrix model}

Throughout this paper we use the same conventions as in ref. [8]. To make the presentation self-contained, we reproduce some of the equations derived in that paper. Details omitted here can be found in [8].

We work with the non-Hermitian chiral random matrix model for the Dirac operator $D$ in the presence of a quark chemical potential introduced by Osborn [10],

$$
D(\mu)=\left(\begin{array}{cc}
0 & i \varphi_{1}+\mu \varphi_{2} \\
i \varphi_{1}^{\dagger}+\mu \varphi_{2}^{\dagger} & 0
\end{array}\right),
$$

where the matrices $\varphi_{1}$ and $\varphi_{2}$ are complex random matrices of dimension $(N+\nu) \times N$. They are distributed according to a Gaussian weight function given by

$$
w(X)=(N / \pi)^{N(N+\nu)} \exp \left(-N \operatorname{tr} X^{\dagger} X\right) .
$$


For a detailed analysis of this model, see also ref. [11]. Since the matrix in eq. (2.1) has $|\nu|$ exact zero modes we can identify $\nu$ with the topological charge. From now on we assume $\nu \geq 0$; the results for $\nu<0$ follow by the replacement $\nu \rightarrow|\nu|$ in the final results. The nonzero eigenvalues of $D(\mu)$ come in $N$ pairs $\left(z_{k},-z_{k}\right)$, and for $\mu=0$ the $z_{k}$ are purely imaginary. Note that $\mu$ in (2.1) is a dimensionless random matrix quantity and should not be confused with the physical chemical potential, see the beginning of section 5 .

For given $\nu$, the partition function of the random matrix model with $N_{f}$ dynamical quarks with masses $m_{f}$ is

$$
Z_{\nu}^{N_{f}}\left(\mu ;\left\{m_{f}\right\}\right)=\int d \varphi_{1} d \varphi_{2} w\left(\varphi_{1}\right) w\left(\varphi_{2}\right) \prod_{f=1}^{N_{f}} \operatorname{det}\left(D(\mu)+m_{f}\right)
$$

with integration measure

$$
d X=\prod_{k=1}^{N+\nu} \prod_{\ell=1}^{N} d \operatorname{Re} X_{k \ell} d \operatorname{Im} X_{k \ell} .
$$

The quenched case corresponds to $N_{f}=0$.

In ref. [10] it was shown that the partition function can be rewritten, up to a normalization constant, as an integral over the eigenvalues $z_{k}$ of $D$,

$$
Z_{\nu}^{N_{f}}\left(\alpha ;\left\{m_{f}\right\}\right)=\int_{\mathbb{C}^{N}}\left[\prod_{k=1}^{N} d^{2} z_{k} w^{\nu}\left(z_{k}, z_{k}^{*} ; \alpha\right) \prod_{f=1}^{N_{f}}\left(m_{f}^{2}-z_{k}^{2}\right)\right]\left|\Delta_{N}\left(\left\{z^{2}\right\}\right)\right|^{2},
$$

where we introduced $\alpha=\mu^{2}$, the integrals over the $z_{k}$ are over the entire complex plane,

$$
\Delta_{N}\left(\left\{z^{2}\right\}\right)=\prod_{k>\ell}\left(z_{k}^{2}-z_{\ell}^{2}\right)
$$

is a Vandermonde determinant, the weight function is given by

$$
w^{\nu}\left(z, z^{*} ; \alpha\right)=|z|^{2 \nu+2} \exp \left(-\frac{N(1-\alpha)}{4 \alpha}\left(z^{2}+z^{* 2}\right)\right) K_{\nu}\left(\frac{N(1+\alpha)}{2 \alpha}|z|^{2}\right),
$$

and $K_{\nu}$ is a modified Bessel function. The orthogonal polynomials corresponding to the weight function (2.7) are [10]

$$
p_{k}^{\nu}(z ; \alpha)=\left(\frac{1-\alpha}{N}\right)^{k} k ! L_{k}^{\nu}\left(-\frac{N z^{2}}{1-\alpha}\right)
$$

where $L_{k}^{\nu}(z)$ is the generalized Laguerre polynomial of order $\nu$ and degree $k$. The corresponding orthogonality relation is

$$
\int_{\mathbb{C}} d^{2} z w^{\nu}\left(z, z^{*} ; \alpha\right) p_{k}^{\nu}(z ; \alpha) p_{\ell}^{\nu}(z ; \alpha)^{*}=r_{k}^{\nu}(\alpha) \delta_{k \ell}
$$

with norm

$$
r_{k}^{\nu}(\alpha)=\frac{\pi \alpha(1+\alpha)^{2 k+\nu} k !(k+\nu) !}{N^{2 k+\nu+2}}
$$


The recurrence relation for the generalized Laguerre polynomials,

$$
(k+1) L_{k+1}^{\nu}(x)=(k+1+\nu) L_{k}^{\nu}(x)-x L_{k}^{\nu+1}(x),
$$

translates into a recurrence relation for the orthogonal polynomials $p_{k}^{\nu}$,

$$
p_{k+1}^{\nu}(z ; \alpha)=(k+1+\nu)\left(\frac{1-\alpha}{N}\right) p_{k}^{\nu}(z ; \alpha)+z^{2} p_{k}^{\nu+1}(z ; \alpha) .
$$

We will also use the Cauchy transform of the orthogonal polynomials defined by

$$
h_{k}^{\nu}(m ; \alpha)=\int_{\mathbb{C}} \frac{d^{2} z}{z^{2}-m^{2}} w^{\nu}\left(z, z^{*} ; \alpha\right) p_{k}^{\nu}(z ; \alpha)^{*} .
$$

The ensemble average of an observable $\mathcal{O}$ is given by

$$
\langle\mathcal{O}\rangle_{\nu, N_{f}}=\frac{1}{Z_{\nu}^{N_{f}}} \int_{\mathbb{C}^{N}}\left[\prod_{k=1}^{N} d^{2} z_{k} w^{\nu}\left(z_{k}, z_{k}^{*} ; \alpha\right) \prod_{f=1}^{N_{f}}\left(m_{f}^{2}-z_{k}^{2}\right)\right]\left|\Delta_{N}\left(\left\{z^{2}\right\}\right)\right|^{2} \mathcal{O}\left(z_{1}, \ldots, z_{N}\right) .
$$

We will frequently omit one or both of the subscripts on $\langle\mathcal{O}\rangle$.

\section{Average phase of the fermion determinant}

Adding a quark mass $m$ to the Dirac operator we define $D(m ; \mu)=D(\mu)+m \mathbb{1}$, where $m$ is assumed to be real. Writing $\operatorname{det} D(m ; \mu)=r e^{i \theta}$, the phase of the determinant follows from $[6]$

$$
e^{2 i \theta}=\frac{\operatorname{det}(D(\mu)+m)}{\operatorname{det}\left(D^{\dagger}(\mu)+m\right)}=\prod_{k=1}^{N} \frac{m^{2}-z_{k}^{2}}{m^{2}-z_{k}^{* 2}} .
$$

Here, $m$ is viewed as a valence quark mass. We are interested in the ensemble average of $e^{2 i \theta}$ with two light dynamical quarks that have the same mass as the valence quark. This quantity is a measure of the fluctuations of the two-flavor determinant in the QCD weight function. For brevity we call $e^{2 i \theta}$ the phase of the determinant, although it really is the phase of the two-flavor determinant. Note that the average phase is real since each matrix appears in the ensemble with the same probability (2.2) as its Hermitian conjugate.

In the presence of $N_{f}$ dynamical quarks the average phase for a valence quark of mass $m$ is given by

$$
\begin{aligned}
\left\langle e^{2 i \theta}\right\rangle_{N_{f}} & =\left\langle\frac{\operatorname{det}(D(\mu)+m)}{\operatorname{det}\left(D^{\dagger}(\mu)+m\right)}\right\rangle_{N_{f}} \\
& =\frac{1}{Z_{\nu}^{N_{f}}\left(\alpha ;\left\{m_{f}\right\}\right)} \int_{\mathbb{C}^{N}}\left[\prod_{k=1}^{N} d^{2} z_{k} w^{\nu}\left(z_{k}, z_{k}^{*} ; \alpha\right) \frac{m^{2}-z_{k}^{2}}{m^{2}-z_{k}^{* 2}} \prod_{f=1}^{N_{f}}\left(m_{f}^{2}-z_{k}^{2}\right)\right]\left|\Delta_{N}\left(\left\{z^{2}\right\}\right)\right|^{2} \\
& =\frac{Z_{\nu}^{N_{f}+1 \mid 1^{*}}\left(\alpha, m ;\left\{m_{f}\right\}\right)}{Z_{\nu}^{N_{f}}\left(\alpha ;\left\{m_{f}\right\}\right)},
\end{aligned}
$$


where $Z_{\nu}^{N_{f}}$ is given by eq. (2.5) and $Z_{\nu}^{N_{f}+1 \mid 1^{*}}$ is the partition function of a random matrix model with $N_{f}+1$ fermionic quarks and one conjugate bosonic quark, see ref. [6] for a detailed discussion. Both partition functions can be interpreted, up to a common additional normalization factor $Z_{\nu}^{0}$, as averages of ratios of characteristic polynomials in the quenched ensemble. Such averages can be computed in terms of the orthogonal polynomials (2.8) and their Cauchy transforms (2.13) using the formalism developed in refs. [12, 13]. The details of its application to eq. (3.2) can be found in ref. [8, section 3.1], and one obtains

$$
\left\langle e^{2 i \theta}\right\rangle_{N_{f}}=\frac{\left|\begin{array}{cccc}
\mathcal{H}_{\nu, 0}(\alpha, m) & \mathcal{H}_{\nu, 1}(\alpha, m) & \cdots & \mathcal{H}_{\nu, N_{f}+1}(\alpha, m) \\
p_{N-1}^{\nu, 0}(m ; \alpha) & p_{N-1}^{\nu, 1}(m ; \alpha) & \cdots & p_{N-1}^{\nu, N_{f}+1}(m ; \alpha) \\
p_{N-1}^{\nu, 0}\left(m_{1} ; \alpha\right) & p_{N-1}^{\nu, 1}\left(m_{1} ; \alpha\right) & \cdots & p_{N-1}^{\nu, N_{f}+1}\left(m_{1} ; \alpha\right) \\
\vdots & \vdots & \vdots & \vdots \\
p_{N-1}^{\nu, 0}\left(m_{N_{f}} ; \alpha\right) & p_{N-1}^{\nu, 1}\left(m_{N_{f}} ; \alpha\right) & \cdots & p_{N-1}^{\nu, N_{f}+1}\left(m_{N_{f}} ; \alpha\right)
\end{array}\right|}{\left[\prod_{f=1}^{N_{f}}\left(m_{f}^{2}-m^{2}\right)\right] \operatorname{det}\left[p_{N}^{\nu, g-1}\left(m_{f} ; \alpha\right)\right]_{f, g=1, \ldots, N_{f}}}
$$

where we introduced the notation

$$
p_{\ell}^{\nu, k}(z ; \alpha)=z^{2 k} p_{\ell}^{\nu+k}(z ; \alpha)
$$

and defined the complex integral

$$
\mathcal{H}_{\nu, k}(\alpha, m)=-\frac{1}{r_{N-1}^{\nu}(\alpha)} \int_{\mathbb{C}} \frac{d^{2} z}{z^{2}-m^{2}} w^{\nu}\left(z, z^{*} ; \alpha\right) p_{N-1}^{\nu, k}\left(z^{*}\right)
$$

over the orthogonal polynomials. In the quenched case (3.3) simplifies to

$$
\left\langle e^{2 i \theta}\right\rangle_{N_{f}=0}=\left|\begin{array}{cc}
\mathcal{H}_{\nu, 0}(\alpha, m) & \mathcal{H}_{\nu, 1}(\alpha, m) \\
p_{N-1}^{\nu, 0}(m ; \alpha) & p_{N-1}^{\nu, 1}(m ; \alpha)
\end{array}\right|
$$

We now consider eq. (3.3) for the special case in which all dynamical fermions have the same mass $m$ as the valence quark. We perform a Taylor expansion of the entries $p_{\ell}^{\nu, k}\left(m_{f}\right)$ of the determinant around $m$,

$$
p_{\ell}^{\nu, k}\left(m_{f} ; \alpha\right)=p_{\ell}^{\nu, k}(m ; \alpha)+\sum_{j=1}^{\infty} \frac{\left(m_{f}-m\right)^{j}}{j !} \partial_{m}^{j} p_{\ell}^{\nu, k}(m ; \alpha), \quad f=1, \ldots, N_{f}
$$

A determinant remains unaltered when linear combinations of its rows are added to any of the rows. Therefore for each additional fermion it is sufficient to keep the next higher-order term in the expansion (3.7). The lower-order terms do not contribute to the determinant since they are identical to the contribution from one of the previous fermions, while the higher-order terms can be neglected since their contribution vanishes for $m_{f} \rightarrow m$. After 
taking each fermion mass in turn to $m$, we obtain

$$
\left\langle e^{2 i \theta}\right\rangle_{N_{f}}=\frac{\left|\begin{array}{cccc}
\mathcal{H}_{\nu, 0}(\alpha, m) & \mathcal{H}_{\nu, 1}(\alpha, m) & \cdots & \mathcal{H}_{\nu, N_{f}+1}(\alpha, m) \\
p_{N-1}^{\nu, 0}(m ; \alpha) & p_{N-1}^{\nu, 1}(m ; \alpha) & \cdots & p_{N-1}^{\nu, N_{f}+1}(m ; \alpha) \\
\partial_{m} p_{N-1}^{\nu, 0}(m ; \alpha) & \partial_{m} p_{N-1}^{\nu, 1}(m ; \alpha) & \cdots & \partial_{m} p_{N-1}^{\nu, N_{f}+1}(m ; \alpha) \\
\vdots & \vdots & \vdots & \vdots \\
\partial_{m}^{N_{f}} p_{N-1}^{\nu, 0}(m ; \alpha) & \partial_{m}^{N_{f}} p_{N-1}^{\nu, 1}(m ; \alpha) & \cdots & \partial_{m}^{N_{f}} p_{N-1}^{\nu, N_{f}+1}(m ; \alpha)
\end{array}\right|}{(2 m)^{N_{f}} N_{f} ! \operatorname{det}\left[\partial_{m}^{f-1} p_{N}^{\nu, g-1}(m ; \alpha)\right]_{f, g=1, \ldots, N_{f}}}
$$

which can also be written in the form

$$
\left\langle e^{2 i \theta}\right\rangle_{N_{f}}=\frac{1}{(2 m)^{N_{f}} N_{f} !} \frac{\mathcal{W}_{N_{f}}^{N-1}(\alpha, m)}{W_{N_{f}}^{N}\left(0,1, \ldots, N_{f}-1\right)},
$$

where

$$
\mathcal{W}_{N_{f}}^{N-1}(\alpha, m)=\sum_{k=0}^{N_{f}+1}(-)^{k} \mathcal{H}_{\nu, k}(\alpha, m) W_{N_{f}+1}^{N-1}\left(0, \ldots, k-1, k+1, \ldots, N_{f}+1\right)
$$

is a sum of Wronskians of order $N_{f}+1$ with indices ranging from 0 to $N_{f}+1$, where in each term a different index $k$ is absent. The Wronskian

$$
W_{n}^{\ell}\left(p_{\ell}^{\nu, k_{1}}(m ; \alpha), \ldots, p_{\ell}^{\nu, k_{n}}(m ; \alpha)\right)=\operatorname{det}\left[\partial_{m}^{i-1} p_{\ell}^{\nu, k_{j}}(m ; \alpha)\right]_{i, j=1, \ldots, n}
$$

in eqs. (3.9) and (3.10) is abbreviated by $W_{n}^{\ell}\left(k_{1}, \ldots, k_{n}\right)$.

\section{Solving the complex Cauchy transform}

The complex integral (3.5) needed in the computation of the phase factor is strongly oscillating and cannot easily be evaluated numerically to high accuracy. In ref. [8] we solved this integral in the microscopic limit using quite involved complex analysis. We introduced integration contours which were then deformed such that the result was composed of contributions from the branch cut discontinuity and from the singularity of the modified $K$-Bessel function. The final result was given in terms of well-behaved one-dimensional integrals plus a short double sum. In this section we present a different derivation, which has the additional advantage of giving a calculable result for finite $N$, away from the microscopic limit.

We first explicitly substitute the weight factor (2.7) in eq. (3.5),

$$
\mathcal{H}_{\nu, k}(\alpha, m)=-\frac{1}{r_{N-1}^{\nu}(\alpha)} \int_{\mathbb{C}} \frac{d^{2} z}{z^{2}-m^{2}}|z|^{2(\nu+1)} e^{-a\left(z^{2}+z^{* 2}\right)} K_{\nu}\left(b|z|^{2}\right) p_{N-1}^{\nu, k}\left(z^{*} ; \alpha\right),
$$

where we defined $a=N(1-\alpha) / 4 \alpha$ and $b=N(1+\alpha) / 2 \alpha$. In ref. [9], Osborn, Splittorff and Verbaarschot solved the Cauchy transform (2.13), which corresponds to the special case of 
$k=0$ in eq. (4.1). ${ }^{1}$ In the following we extend their result to arbitrary positive integer $k$, as required in eq. (3.3). Following the derivation in section $\mathrm{V}$ of ref. [9] we write

$$
\frac{e^{-a z^{2}}}{z^{2}-m^{2}}=\frac{e^{-a m^{2}}}{z^{2}-m^{2}}+\frac{e^{-a z^{2}}-e^{-a m^{2}}}{z^{2}-m^{2}}
$$

and decompose eq. (4.1) accordingly as $\mathcal{H}_{\nu, k}=\mathcal{H}_{\nu, k}^{(1)}+\mathcal{H}_{\nu, k}^{(2)}$ with

$$
\begin{aligned}
\mathcal{H}_{\nu, k}^{(1)}(\alpha, m) & =-\frac{e^{-a m^{2}}}{r_{N-1}^{\nu}(\alpha)} \int_{\mathbb{C}} \frac{d^{2} z}{z^{2}-m^{2}}|z|^{2(\nu+1)} e^{-a z^{* 2}} K_{\nu}\left(b|z|^{2}\right) p_{N-1}^{\nu, k}\left(z^{*} ; \alpha\right), \\
\mathcal{H}_{\nu, k}^{(2)}(\alpha, m) & =-\frac{1}{r_{N-1}^{\nu}(\alpha)} \int_{\mathbb{C}} d^{2} z|z|^{2(\nu+1)} \frac{e^{-a z^{2}}-e^{-a m^{2}}}{z^{2}-m^{2}} e^{-a z^{* 2}} K_{\nu}\left(b|z|^{2}\right) p_{N-1}^{\nu, k}\left(z^{*} ; \alpha\right) .
\end{aligned}
$$

For $\mathcal{H}_{\nu, k}^{(1)}$ we consider the cases $|z|<m$ and $|z|>m$ separately and expand the denominator in a geometric series,

$$
\begin{aligned}
\mathcal{H}_{\nu, k}^{(1)}(\alpha, m)=- & \frac{e^{-a m^{2}}}{r_{N-1}^{\nu}(\alpha)}\left[-\sum_{j=0}^{\infty} \frac{1}{m^{2(j+1)}} \int_{|z|<m} d^{2} z|z|^{2(\nu+1)} K_{\nu}\left(b|z|^{2}\right) z^{2 j} e^{-a z^{* 2}} p_{N-1}^{\nu, k}\left(z^{*} ; \alpha\right)\right. \\
& \left.+\sum_{j=0}^{\infty} m^{2 j} \int_{|z|>m} d^{2} z|z|^{2(\nu+1)} K_{\nu}\left(b|z|^{2}\right) \frac{1}{z^{2(j+1)}} e^{-a z^{* 2}} p_{N-1}^{\nu, k}\left(z^{*} ; \alpha\right)\right] .
\end{aligned}
$$

The polynomials $p_{\ell}^{\nu, k}(z ; \alpha)$ defined in (3.4) are even in their argument $z$ so that we can implicitly define the expansion

$$
f\left(z^{2}\right)=e^{-a z^{2}} p_{N-1}^{\nu, k}(z ; \alpha)=\sum_{n=0}^{\infty} a_{n} z^{2 n} .
$$

In polar coordinates the angular part of the integrands in eq. (4.5) can therefore be written as a product of power series in $z^{2}$ and $z^{* 2}$. With $z=r e^{i \theta}$, the angular integration of such a product can be computed analytically using

$$
\int_{0}^{2 \pi} d \theta z^{2 k} z^{* 2 \ell}=2 \pi r^{4 k} \delta_{k \ell}
$$

After angular integration the second integral in eq. (4.5) vanishes, while the first one gets contributions

$$
\int_{0}^{2 \pi} d \theta z^{2 j} f\left(z^{* 2}\right)=\sum_{n=0}^{\infty} a_{n} \int_{0}^{2 \pi} d \theta z^{2 j} z^{* 2 n}=2 \pi a_{j} r^{4 j} .
$$

Resumming the $a_{j}$ using eq. (4.6), the integral (4.5) can then be written as

$$
\begin{aligned}
\mathcal{H}_{\nu, k}^{(1)}(\alpha, m) & =\frac{2 \pi e^{-a m^{2}}}{m^{2} r_{N-1}^{\nu}(\alpha)} \int_{0}^{m} d r r^{2 \nu+3} K_{\nu}\left(b r^{2}\right) f\left(\frac{r^{4}}{m^{2}}\right) \\
& =\frac{\pi m^{\nu} e^{-a m^{2}}}{r_{N-1}^{\nu}(\alpha)} \int_{0}^{m} d u u^{\nu+1} K_{\nu}(b m u) e^{-a u^{2}} p_{N-1}^{\nu, k}(u ; \alpha),
\end{aligned}
$$

\footnotetext{
${ }^{1}$ We thank Jac Verbaarschot for drawing our attention to the solution of the Cauchy transform in ref. [9].
} 
where in the last step we have introduced the variable transformation $u=r^{2} / m$ and replaced $f\left(u^{2}\right)$ by its explicit expression (4.6).

We now turn to $\mathcal{H}_{\nu, k}^{(2)}$ given by eq. (4.4). The second term on the RHS of eq. (4.2) is analytic and can be expanded as

$$
\frac{e^{-a z^{2}}-e^{-a m^{2}}}{z^{2}-m^{2}}=e^{-a z^{2}} \sum_{n=0}^{\infty} d_{n} p_{n}^{\nu}(z ; \alpha),
$$

where the coefficients $d_{n}$ were computed in ref. [9] and are given by

$$
d_{n}=-\frac{N^{n+1}}{n !(1-\alpha)^{n+1}} \int_{0}^{(1-\alpha)^{2} / 4 \alpha} d t e^{-m^{2} N t /(1-\alpha)} \frac{t^{n}}{(t+1)^{n+\nu+1}} .
$$

Note that the explicit factor $e^{-a z^{2}}$ in eq. (4.10) is such that the weight of the orthogonal polynomials are retrieved after substituting eq. (4.10) in eq. (4.4). This yields

$$
\mathcal{H}_{\nu, k}^{(2)}(\alpha, m)=-\frac{1}{r_{N-1}^{\nu}(\alpha)} \sum_{n=0}^{\infty} d_{n} \int_{\mathbb{C}} d^{2} z w^{\nu}\left(z, z^{*} ; \alpha\right) p_{n}^{\nu}(z ; \alpha) p_{N-1}^{\nu, k}\left(z^{*} ; \alpha\right) .
$$

In ref. [9] the integral (4.12) was solved for $k=0$. In that case the solution immediately follows from the orthogonality relation (2.9), resulting in

$$
\mathcal{H}_{\nu, 0}^{(2)}(\alpha, m)=-d_{N-1}=\frac{N^{N}}{(N-1) !(1-\alpha)^{N}} \int_{0}^{(1-\alpha)^{2} / 4 \alpha} d t e^{-m^{2} N t /(1-\alpha)} \frac{t^{N-1}}{(t+1)^{N+\nu}},
$$

where we substituted the explicit expression for $d_{N-1}$ given in eq. (4.11). To compute the phase of the fermion determinant for arbitrary $N_{f}$ and $\nu$ we also need the solution of eq. (4.12) for positive integer $k$. In this case the second polynomial in the weighted integral is $p_{N-1}^{\nu, k}\left(z^{*} ; \alpha\right)$, which involves an orthogonal polynomial of order $\nu+k$ instead of $\nu$ and an additional power of $z^{*}$, such that the orthogonality relation (2.9) can no longer be applied directly. To solve the integral (4.12) for arbitrary $k \in \mathbb{N}$ we use the relation

$$
p_{\ell}^{\nu, k}(z ; \alpha)=z^{2 k} p_{\ell}^{\nu+k}(z ; \alpha)=\sum_{j=0}^{k}\left(\begin{array}{c}
k \\
j
\end{array}\right) \frac{(\ell+\nu+k) !}{(\ell+\nu+k-j) !}\left(\frac{\alpha-1}{N}\right)^{j} p_{\ell+k-j}^{\nu}(z ; \alpha)
$$

for any $\nu, \ell, k \in \mathbb{N}$, which expresses the LHS as a sum over orthogonal polynomials of order $\nu$ and degree $\ell, \ldots, \ell+k$. The proof of this relation is given in appendix A. We now substitute this expansion in eq. (4.12) to find

$$
\begin{aligned}
\mathcal{H}_{\nu, k}^{(2)}(\alpha, m)= & -\frac{1}{r_{N-1}^{\nu}(\alpha)} \sum_{j=0}^{k}\left(\begin{array}{l}
k \\
j
\end{array}\right) \frac{(N-1+\nu+k) !}{(N-1+\nu+k-j) !}\left(\frac{\alpha-1}{N}\right)^{j} \\
& \times \sum_{n=0}^{\infty} d_{n} \int_{\mathbb{C}} d^{2} z w^{\nu}\left(z, z^{*} ; \alpha\right) p_{n}^{\nu}(z ; \alpha) p_{N-1+k-j}^{\nu}\left(z^{*} ; \alpha\right) \\
= & -\frac{1}{r_{N-1}^{\nu}(\alpha)} \sum_{j=0}^{k}\left(\begin{array}{l}
k \\
j
\end{array}\right) \frac{(N-1+\nu+k) !}{(N-1+\nu+k-j) !}\left(\frac{\alpha-1}{N}\right)^{j} d_{N-1+k-j} r_{N-1+k-j}^{\nu}(\alpha),
\end{aligned}
$$


where in the last step we have applied the orthogonality relation (2.9), after which only the $n=N-1+k-j$ term survives. Using the norm (2.10) we compute the ratio

$$
\frac{r_{N-1+k-j}^{\nu}(\alpha)}{r_{N-1}^{\nu}(\alpha)}=\frac{(1+\alpha)^{2(k-j)}(N-1+k-j) !(N-1+k-j+\nu) !}{N^{2(k-j)}(N-1) !(N-1+\nu) !}
$$

and after substituting this and the expression (4.11) for $d$ in eq. (4.15) we find

$$
\begin{aligned}
\mathcal{H}_{\nu, k}^{(2)}(\alpha, m)= & \frac{N^{N-k}}{(1-\alpha)^{N+k}} \frac{(N-1+\nu+k) !}{(N-1) !(N-1+\nu) !} \int_{0}^{(1-\alpha)^{2} / 4 \alpha} d t e^{-m^{2} N t /(1-\alpha)} \frac{t^{N-1}}{(t+1)^{N+k+\nu}} \\
& \times \sum_{j=0}^{k}(-)^{j}\left(\begin{array}{c}
k \\
j
\end{array}\right)(1+\alpha)^{2(k-j)} t^{k-j}(1-\alpha)^{2 j}(t+1)^{j} \\
= & N^{N-k}(4 \alpha)^{k+\nu}(1-\alpha)^{N+k} \frac{(N-1+\nu+k) !}{(N-1) !(N-1+\nu) !} \\
& \times \int_{0}^{1} d u e^{-m^{2} a u} \frac{u^{N-1}(u-1)^{k}}{\left[(1-\alpha)^{2} u+4 \alpha\right]^{N+k+\nu}} .
\end{aligned}
$$

In the last step we have performed the sum over $j$ using the binomial theorem to obtain $\left[4 \alpha t-(1-\alpha)^{2}\right]^{k}$. Also, we have introduced the variable transformation $u=4 \alpha t /(1-\alpha)^{2}$ to simplify the integration range.

The sum of $\mathcal{H}_{\nu, k}^{(1)}$ and $\mathcal{H}_{\nu, k}^{(2)}$ of eqs. (4.9) and (4.17) gives the exact finite- $N$ result for $\mathcal{H}_{\nu, k}$ needed to compute the average phase of the fermion determinant with eqs. (3.3), (3.6) and (3.9). For $\nu<0$ one just needs to replace $\nu$ by $|\nu|$. Note that the chiral limit $(m \rightarrow 0)$ of the phase factor (3.9) has to be taken carefully, as detailed in appendix B.1.

\section{Microscopic limit}

The results computed from chRMT are universal, i.e., identical to the corresponding quantities in QCD, in the so-called microscopic regime. This regime is obtained by defining the rescaled parameters $\hat{m}=2 N m, \hat{m}_{f}=2 N m_{f}, \hat{\alpha}=2 N \alpha$ and the rescaled eigenvalues $\hat{z}=2 N z$ and then taking $N \rightarrow \infty$ while keeping the rescaled quantities fixed. The conversion of the rescaled random matrix parameters to the physical parameters is done using the relations $\hat{z}=z_{\text {phys }} V \Sigma, \hat{m}=m_{\text {phys }} V \Sigma$ and $\hat{\alpha}=\hat{\mu}^{2}=\mu_{\text {phys }}^{2} F^{2} V$, where $V$ is the four-volume and $\Sigma$ and $F$ are low-energy constants of chiral perturbation theory. The Gell-Mann-Oakes-Renner relation $m_{\pi}^{2} F^{2}=2 m \Sigma$ (for equal quark masses) can be used to introduce the physical pion mass $m_{\pi}$ through the combination $\mu_{\text {phys }}^{2} / m_{\pi}^{2}=\hat{\mu}^{2} / 2 \hat{m}$.

To take the microscopic limit of the average phase we introduce the corresponding limits of the various objects defined in section 2 and section 3. A detailed derivation of these limits can be found in ref. [8, appendix A]. The microscopic limit (denoted by a subscript $s$ ) of the orthogonal polynomials (2.8) is defined as

$$
p_{s}^{\nu}(\hat{z} ; \hat{\alpha})=\lim _{N \rightarrow \infty} \frac{e^{N}}{(2 N)^{\nu+1 / 2}} p_{N-1}^{\nu}(\hat{z} / 2 N ; \hat{\alpha} / 2 N)=\sqrt{\pi} e^{-\hat{\alpha} / 2} \hat{z}^{-\nu} I_{\nu}(\hat{z}) .
$$


Accordingly, the microscopic limit of $p_{N-1}^{\nu, k}$, defined in eq. (3.4), is

$$
p_{s}^{\nu, k}(\hat{z} ; \hat{\alpha})=\lim _{N \rightarrow \infty} \frac{e^{N}}{(2 N)^{\nu-k+1 / 2}} p_{N-1}^{\nu, k}(\hat{z} / 2 N ; \hat{\alpha} / 2 N)=\sqrt{\pi} e^{-\hat{\alpha} / 2} \hat{z}^{-\nu} I_{\nu, k}(\hat{z})
$$

where we introduced the notation

$$
I_{\nu, k}(z)=z^{k} I_{\nu+k}(z)
$$

The microscopic limit of the weight function (2.7) is

$$
w_{s}^{\nu}\left(\hat{z}, \hat{z}^{*} ; \hat{\alpha}\right)=\lim _{N \rightarrow \infty}(2 N)^{2 \nu+2} w^{\nu}\left(\hat{z} / 2 N, \hat{z}^{*} / 2 N ; \hat{\alpha} / 2 N\right)=|\hat{z}|^{2(\nu+1)} e^{-\frac{\hat{z}^{2}+\hat{z}^{* 2}}{8 \hat{\alpha}}} K_{\nu}\left(\frac{|\hat{z}|^{2}}{4 \hat{\alpha}}\right)
$$

and the microscopic limit of the normalization factor (2.10) is

$$
r_{s}^{\nu}(\hat{\alpha})=\lim _{N \rightarrow \infty}(2 N)^{2} e^{2 N} r_{N-1}^{\nu}(\hat{\alpha} / 2 N)=4 \pi^{2} \hat{\alpha} e^{\hat{\alpha}} .
$$

We define the microscopic limit of the integral (3.5) as

$$
\mathcal{H}_{\nu, k}^{s}(\hat{\alpha}, \hat{m})=\lim _{N \rightarrow \infty} e^{-N}(2 N)^{\nu+k-1 / 2} \sqrt{\pi} e^{-\hat{\alpha} / 2} \hat{m}^{-\nu} \mathcal{H}_{\nu, k}(\hat{\alpha} / 2 N, \hat{m} / 2 N)
$$

where the prefactors were chosen such that it coincides with the master integral of ref. [8, eq. (3.21)] and remains finite when $N \rightarrow \infty$, i.e.,

$$
\mathcal{H}_{\nu, k}^{s}(\hat{\alpha}, \hat{m})=-\frac{e^{-2 \hat{\alpha}}}{4 \pi \hat{\alpha} \hat{m}^{\nu}} \int_{\mathbb{C}} \frac{d^{2} z}{z^{2}-\hat{m}^{2}} \frac{|z|^{2(\nu+1)}}{z^{* \nu}} e^{-\frac{z^{2}+z^{* 2}}{8 \hat{\alpha}}} K_{\nu}\left(\frac{\left|z^{2}\right|}{4 \hat{\alpha}}\right) I_{\nu, k}\left(z^{*}\right) .
$$

Here we also used the microscopic limits (5.2), (5.4) and (5.5). Using eqs. (5.2) and (5.6) we now construct the microscopic limit of the phase factor derived in section 3. For the unquenched case we take the microscopic limit of eq. (3.3) and find

$$
\left\langle e_{s}^{2 i \theta}\right\rangle_{N_{f}}=\lim _{N \rightarrow \infty}\left\langle e^{2 i \theta}\right\rangle_{N_{f}}=\frac{\left|\begin{array}{cccc}
\mathcal{H}_{\nu, 0}^{s}(\hat{\alpha}, \hat{m}) & \mathcal{H}_{\nu, 1}^{s}(\hat{\alpha}, \hat{m}) & \cdots & \mathcal{H}_{\nu, N_{f}+1}^{s}(\hat{\alpha}, \hat{m}) \\
I_{\nu, 0}(\hat{m}) & I_{\nu, 1}(\hat{m}) & \cdots & I_{\nu, N_{f}+1}(\hat{m}) \\
I_{\nu, 0}\left(\hat{m}_{1}\right) & I_{\nu, 1}\left(\hat{m}_{1}\right) & \cdots & I_{\nu, N_{f}+1}\left(\hat{m}_{1}\right) \\
\vdots & \vdots & \vdots & \vdots \\
I_{\nu, 0}\left(\hat{m}_{N_{f}}\right) & I_{\nu, 1}\left(\hat{m}_{N_{f}}\right) & \cdots & I_{\nu, N_{f}+1}\left(\hat{m}_{N_{f}}\right)
\end{array}\right|}{\left[\prod_{f=1}^{N_{f}}\left(\hat{m}_{f}^{2}-\hat{m}^{2}\right)\right] \operatorname{det}\left[I_{\nu, g-1}\left(\hat{m}_{f}\right)\right]_{f, g=1, \ldots, N_{f}}} .
$$

As expected, the dependence on $N$ has dropped out, leaving a finite microscopic limit for the average phase factor. Similarly, the microscopic limit of the quenched average phase factor (3.6) is given by

$$
\left\langle e_{s}^{2 i \theta}\right\rangle_{N_{f}=0}=\lim _{N \rightarrow \infty}\left\langle e^{2 i \theta}\right\rangle_{N_{f}=0}=\left|\begin{array}{cc}
\mathcal{H}_{\nu, 0}^{s}(\hat{\alpha}, \hat{m}) & \mathcal{H}_{\nu, 1}^{s}(\hat{\alpha}, \hat{m}) \\
I_{\nu, 0}(\hat{m}) & I_{\nu, 1}(\hat{m})
\end{array}\right| .
$$


For the special case in which all dynamical fermions have the same mass $\hat{m}$ as the valence quark, eq. (5.8) becomes

$$
\left\langle e_{s}^{2 i \theta}\right\rangle_{N_{f}}=\frac{\left|\begin{array}{cccc}
\mathcal{H}_{\nu, 0}^{s}(\hat{\alpha}, \hat{m}) & \mathcal{H}_{\nu, 1}^{s}(\hat{\alpha}, \hat{m}) & \cdots & \mathcal{H}_{\nu, N_{f}+1}^{s}(\hat{\alpha}, \hat{m}) \\
I_{\nu, 0}(\hat{m}) & I_{\nu, 1}(\hat{m}) & \cdots & I_{\nu, N_{f}+1}(\hat{m}) \\
I_{\nu, 0}^{\prime}(\hat{m}) & I_{\nu, 1}^{\prime}(\hat{m}) & \cdots & I_{\nu, N_{f}+1}^{\prime}(\hat{m}) \\
\vdots & \vdots & \vdots & \vdots \\
I_{\nu, 0}^{\left(N_{f}\right)}(\hat{m}) & I_{\nu, 1}^{\left(N_{f}\right)}(\hat{m}) & \cdots & I_{\nu, N_{f}+1}^{\left(N_{f}\right)}(\hat{m})
\end{array}\right|}{(2 \hat{m})^{N_{f}} N_{f} ! \operatorname{det}\left[I_{\nu, g-1}^{(f-1)}(\hat{m})\right]_{f, g=1, \ldots, N_{f}}}
$$

in analogy to section 3. Again, an alternative way to write this result is

$$
\left\langle e_{s}^{2 i \theta}\right\rangle_{N_{f}}=\frac{1}{(2 \hat{m})^{N_{f}} N_{f} !} \frac{\mathcal{W}_{N_{f}}(\hat{\alpha}, \hat{m})}{W_{N_{f}}\left(0,1, \ldots, N_{f}-1\right)},
$$

where we have defined

$$
\mathcal{W}_{N_{f}}(\hat{\alpha}, \hat{m})=\sum_{k=0}^{N_{f}+1}(-)^{k} \mathcal{H}_{\nu, k}^{s}(\hat{\alpha}, \hat{m}) W_{N_{f}+1}\left(0, \ldots, k-1, k+1, \ldots, N_{f}+1\right)
$$

in analogy to $(3.10)$ and $W_{n}\left(k_{1}, \ldots, k_{n}\right)$ is a short-hand notation for the Wronskian

$$
W_{n}\left(I_{\nu, k_{1}}(\hat{m}), \ldots, I_{\nu, k_{n}}(\hat{m})\right)=\operatorname{det}\left[I_{\nu, k_{j}}^{(i-1)}(\hat{m})\right]_{i, j=1, \ldots, n}
$$

appearing in eqs. (5.11) and (5.12).

To compute the microscopic limit (5.8) of the average phase we need to take the microscopic limit (5.6) of the complex integral (3.5), which was solved for finite $N$ by eqs. (4.9) and (4.17). Taking the microscopic limit (5.6) of eqs. (4.9) and (4.17) gives

$$
\begin{aligned}
\mathcal{H}_{\nu, k}^{s}(\hat{\alpha}, \hat{m})= & \frac{e^{-2 \hat{\alpha}-\frac{\hat{m}^{2}}{8 \hat{\alpha}}}}{4 \hat{\alpha}} \int_{0}^{\hat{m}} d u u^{k+1} e^{-\frac{u^{2}}{8 \hat{\alpha}}} K_{\nu}\left(\frac{\hat{m} u}{4 \hat{\alpha}}\right) I_{\nu+k}(u) \\
& +\frac{(4 \hat{\alpha})^{\nu+k}}{2 \hat{m}^{\nu}} \int_{0}^{1} d u e^{-\frac{2 \hat{\alpha}}{u}-\frac{\hat{m}^{2} u}{8 \hat{\alpha}}} \frac{(u-1)^{k}}{u^{\nu+k+1}}
\end{aligned}
$$

where in the first term we have substituted eqs. (5.2) and (5.5) and in the second term we have used the definition

$$
\lim _{N \rightarrow \infty}\left(1-\frac{\hat{\alpha}}{N}\right)^{N}=e^{-\hat{\alpha}}
$$

of the exponential function and Stirling's formula

$$
\lim _{N \rightarrow \infty} \frac{N !}{\sqrt{2 \pi N} N^{N} e^{-N}}=1 .
$$

For the special case of $k=0$, eq. (5.14) reproduces the result which was computed previously in ref. [9] in a study of the chiral condensate. However, for the calculation of the 
average phase using eq. (5.8) we need the more general result of eq. (5.14) for arbitrary $k \in \mathbb{N}$. The chiral limit of the phase factor (5.11) is computed in appendix B.2.

In ref. [8] we computed the complex integral (5.7) in a different way, which led to an integral representation that looks quite different from eq. (5.14). In the next section we will prove the equivalence of the expressions derived in both formulations.

Apart from providing an alternative formula to compute the microscopic limit of the average phase, the current derivation has the significant additional feature that it also gives a calculable and well-behaved expression for finite-sized matrices, away from the microscopic limit, given by the sum of eqs. (4.9) and (4.17). This expression will be useful when verifying the analytical RMT results by dynamical RMT simulations in section 7 .

\section{Equivalence of integration results}

In ref. [8] the complex integral (5.7) was solved using a completely different formalism, based on the deformation of integration contours and some involved complex analysis, leading to

$$
\begin{aligned}
\mathcal{H}_{\nu, k}^{s}(\hat{\alpha}, \hat{m})= & \frac{e^{-2 \hat{\alpha}-\frac{\hat{m}^{2}}{8 \hat{\alpha}}}}{4 \hat{\alpha}}\left[\int_{0}^{\hat{m}} d u u^{k+1} e^{-\frac{u^{2}}{8 \hat{\alpha}}} K_{\nu}\left(\frac{\hat{m} u}{4 \hat{\alpha}}\right) I_{\nu+k}(u)\right. \\
& \left.+\int_{0}^{\infty} d u(-)^{k} u^{k+1} e^{-\frac{u^{2}}{8 \hat{\alpha}}} I_{\nu}\left(\frac{\hat{m} u}{4 \hat{\alpha}}\right) K_{\nu+k}(u)\right] \\
& +e^{-2 \hat{\alpha}-\frac{\hat{m}^{2}}{8 \hat{\alpha}}} \frac{(-)^{k} 2^{\nu-1+k}}{\hat{m}^{\nu}} \sum_{i, j=0}^{i+j \leq \nu-1} \frac{(\nu-1-i) !(\nu-1+k-j) !}{(\nu-1-i-j) ! i ! j !}\left(\frac{\hat{m}^{2}}{8 \hat{\alpha}}\right)^{i}(2 \hat{\alpha})^{j} .
\end{aligned}
$$

This solution must be equivalent to (5.14), as they are just two different representations of the same complex integral (5.7). Nevertheless, as both expressions look quite different it is useful to prove their equivalence, without resorting to the complicated complex analysis used in ref. [8]. This proof will also provide a check on both results.

The first integral in eq. (5.14) is identical to the first integral in eq. (6.1). Therefore it remains to show that the second integral in eq. (5.14) equals the sum of the second integral and the additional polynomial in eq. (6.1). Setting $a=2 \hat{\alpha}$ and $b=\hat{m}^{2} / 8 \hat{\alpha}$ to simplify the notation and canceling some prefactors, we thus need to show

$a^{\nu+k} \int_{0}^{1} d u e^{-\frac{a}{u}-b u} \frac{(1-u)^{k}}{u^{\nu+k+1}} \stackrel{?}{=} e^{-a-b}\left[\frac{(a b)^{\nu / 2}}{2^{k} a} \int_{0}^{\infty} d u u^{k+1} e^{-\frac{u^{2}}{4 a}} I_{\nu}(u \sqrt{b / a}) K_{\nu+k}(u)+S_{\nu, k}\right]$,

where we have defined

$$
S_{\nu, k}=\sum_{i, j=0}^{i+j \leq \nu-1} \frac{(\nu-1-i) !(\nu-1+k-j) !}{(\nu-1-i-j) ! i ! j !} b^{i} a^{j} .
$$

For the special case of $\nu=k=0$ this was proven in ref. [9]. Below we will give a general proof for arbitrary $\nu$ and $k$. 
In appendix $\mathrm{C}$ we prove the integral relation

$$
\begin{aligned}
\int_{0}^{\infty} d u & u^{k+1} e^{-\frac{u^{2}}{4 a}} I_{\nu}(u \sqrt{b / a}) K_{\nu+k}(u) \\
& =2^{k} k ! a\left(\frac{b}{a}\right)^{\nu / 2} e^{a+b} \int_{0}^{1} d s e^{-\frac{a}{s}-b s} s^{\nu+k-1} L_{k}^{\nu}(-b(1-s)) .
\end{aligned}
$$

Renaming $u$ to $s$ on the LHS of eq. (6.2) and substituting (6.4) in the first term on the RHS, proving (6.2) corresponds to proving the identity

$$
\mathcal{J}_{\nu, k} \equiv k ! b^{\nu} \int_{0}^{1} d s e^{-\frac{a}{s}-b s} s^{\nu+k-1} L_{k}^{\nu}(-b(1-s)) \stackrel{?}{=} a^{\nu+k} \int_{0}^{1} d s e^{-\frac{a}{s}-b s} \frac{(1-s)^{k}}{s^{\nu+k+1}}-e^{-a-b} S_{\nu, k} .
$$

Using the Rodrigues formula for the generalized Laguerre polynomials,

$$
L_{k}^{\nu}(x)=\frac{e^{x} x^{-\nu}}{k !} \frac{d^{k}}{d x^{k}}\left(e^{-x} x^{k+\nu}\right),
$$

the LHS of eq. (6.5) can be written as

$$
\begin{aligned}
\mathcal{J}_{\nu, k} & =(-)^{k} b^{\nu} \int_{0}^{1} d s e^{-\frac{a}{s}} \frac{s^{\nu+k-1}}{(1-s)^{\nu}} \frac{d^{k}}{d s^{k}}\left[e^{-b s}(1-s)^{k+\nu}\right] \\
& =b^{\nu} \int_{0}^{1} d s \frac{d^{k}}{d s^{k}}\left[e^{-\frac{a}{s}} \frac{s^{\nu+k-1}}{(1-s)^{\nu}}\right] e^{-b s}(1-s)^{k+\nu}
\end{aligned}
$$

where in the last step we have performed $k$ successive integrations by parts. In appendix D we prove that

$$
\frac{d^{k}}{d s^{k}}\left[e^{-\frac{a}{s}} \frac{s^{\nu+k-1}}{(1-s)^{\nu}}\right]=e^{-\frac{a}{s}} \sum_{j=0}^{k}\left(\begin{array}{c}
k \\
j
\end{array}\right) \frac{(\nu)_{j} a^{k-j} s^{\nu-k+j-1}}{(1-s)^{\nu+j}},
$$

with the Pochhammer symbol $(\nu)_{j}$ defined in appendix $\mathrm{C}$, and substituting this in the integral (6.7) we obtain

$$
\mathcal{J}_{\nu, k}=b^{\nu} \int_{0}^{1} d s e^{-\frac{a}{s}-b s} \sum_{j=0}^{k}\left(\begin{array}{c}
k \\
j
\end{array}\right)(\nu)_{j} a^{k-j} s^{\nu-k+j-1}(1-s)^{k-j} .
$$

With this identity we show in appendix $\mathrm{E}$ that $\mathcal{J}_{\nu, k}$ satisfies the recurrence relation

$$
\mathcal{J}_{\nu, k}=-b^{\nu-1} e^{-a-b}(\nu)_{k}+\sum_{j=0}^{k}(j+1)_{k-j}\left[a b \mathcal{J}_{\nu-2, j}+(\nu-1) \mathcal{J}_{\nu-1, j}\right] .
$$

We now prove the equivalence (6.5) by induction in $\nu$ using the recurrence relation (6.10) and the expression (6.9) for $\mathcal{J}_{\nu, k}$. For $\nu=0$ only the $j=0$ term contributes in eq. (6.9), and we find

$$
\mathcal{J}_{0, k}=a^{k} \int_{0}^{1} d s e^{-\frac{a}{s}-b s} s^{-k-1}(1-s)^{k}
$$


which corresponds to the RHS of eq. (6.5) since $S_{0, k}=0$. For $\nu=1$ eq. (6.9) gives

$$
\begin{aligned}
\mathcal{J}_{1, k} & =-\int_{0}^{1} d s\left[e^{-b s}\right]^{\prime} e^{-\frac{a}{s}} \sum_{j=0}^{k}\left(\begin{array}{c}
k \\
j
\end{array}\right) j ! a^{k-j}\left(\frac{1}{s}-1\right)^{k-j} \\
& =-e^{-a-b} k !+\int_{0}^{1} d s e^{-\frac{a}{s}-b s} \sum_{j=0}^{k} \frac{k !}{(k-j) !} a^{k-j}\left(\frac{1}{s}-1\right)^{k-j}\left[\frac{a}{s^{2}}-\frac{k-j}{s(1-s)}\right] \\
& =-e^{-a-b} k !+\int_{0}^{1} d s e^{-\frac{a}{s}-b s}\left[\sum_{j=0}^{k}-\sum_{j=1}^{k}\right] \frac{k !}{(k-j) !} a^{k-j+1} \frac{(1-s)^{k-j}}{s^{k-j+2}} \\
& =-e^{-a-b} k !+a^{k+1} \int_{0}^{1} d s e^{-\frac{a}{s}-b s} \frac{(1-s)^{k}}{s^{k+2}}
\end{aligned}
$$

where in the second step we have integrated by parts and in the third step we have observed that the $j=k$ contribution in the last term vanishes and shifted $j \rightarrow j-1$ for this term so that in the last step only the $j=0$ term of the first sum remains. For $\nu=1$ eq. (6.3) gives $S_{1, k}=k$ ! so that eq. (6.5) is satisfied for $\nu=1$. We now prove that the equivalence holds for $\mathcal{J}_{\nu, k}$ if it is satisfied for $\mathcal{J}_{\nu-1, k}$ and $\mathcal{J}_{\nu-2, k}$. We start from the recurrence relation (6.10) and substitute the RHS of eq. (6.5) for $\nu-1$ and $\nu-2$,

$$
\begin{aligned}
\mathcal{J}_{\nu, k}= & \sum_{j=0}^{k} a^{\nu-1+j}(j+1)_{k-j} \int_{0}^{1} d s e^{-\frac{a}{s}-b s} \frac{(1-s)^{j}}{s^{\nu+j}}[b s+(\nu-1)] \\
& -e^{-a-b}\left\{\sum_{j=0}^{k}(j+1)_{k-j}\left[a b S_{\nu-2, j}+(\nu-1) S_{\nu-1, j}\right]+b^{\nu-1}(\nu)_{k}\right\} .
\end{aligned}
$$

Performing an integration by parts on the first term in the integral yields

$-\int_{0}^{1} d s\left[e^{-b s}\right]^{\prime} e^{-\frac{a}{s}} \frac{(1-s)^{j}}{s^{\nu+j-1}}=\int_{0}^{1} d s e^{-\frac{a}{s}-b s} \frac{(1-s)^{j}}{s^{\nu+j-1}}\left[\frac{a}{s^{2}}-\frac{j}{1-s}-\frac{\nu+j-1}{s}\right]-e^{-a-b} \delta_{j 0}$,

where we observed that the surface term is only nonzero for $j=0$. Let us denote the first term on the RHS of eq. (6.13) by $\mathcal{J}_{\nu, k}^{(1)}$. Substituting (6.14) into this term we obtain

$$
\begin{aligned}
\mathcal{J}_{\nu, k}^{(1)} & =\sum_{j=0}^{k} a^{\nu-1+j}(j+1)_{k-j} \int_{0}^{1} d s e^{-\frac{a}{s}-b s} \frac{(1-s)^{j}}{s^{\nu+j-1}}\left[\frac{a}{s^{2}}-\frac{j}{s(1-s)}\right]-e^{-a-b} a^{\nu-1} k ! \\
& =\int_{0}^{1} d s e^{-\frac{a}{s}-b s}\left[\sum_{j=0}^{k}-\sum_{j=0}^{k-1}\right] a^{\nu+j}(j+1)_{k-j} \frac{(1-s)^{j}}{s^{\nu+j+1}}-e^{-a-b} a^{\nu-1} k ! \\
& =a^{\nu+k} \int_{0}^{1} d s e^{-\frac{a}{s}-b s} \frac{(1-s)^{k}}{s^{\nu+k+1}}-e^{-a-b} a^{\nu-1} k !
\end{aligned}
$$

In the first line, we observed that the $j=0$ contribution to the second term in square brackets vanishes, which allowed us to shift $j \rightarrow j+1$ in the corresponding sum in the 
second line. In the third line only the $j=k$ term of the first sum survives. Putting everything together, we have

$$
\begin{aligned}
\mathcal{J}_{\nu, k}= & a^{\nu+k} \int_{0}^{1} d s e^{-\frac{a}{s}-b s} \frac{(1-s)^{k}}{s^{\nu+k+1}} \\
& -e^{-a-b}\left\{\sum_{j=0}^{k}(j+1)_{k-j}\left[a b S_{\nu-2, j}+(\nu-1) S_{\nu-1, j}\right]+b^{\nu-1}(\nu)_{k}+a^{\nu-1} k !\right\} .
\end{aligned}
$$

To reproduce the RHS of eq. (6.5) it remains to show that

$$
\sum_{j=0}^{k}(j+1)_{k-j}\left[a b S_{\nu-2, j}+(\nu-1) S_{\nu-1, j}\right]+b^{\nu-1}(\nu)_{k}+a^{\nu-1} k ! \stackrel{?}{=} S_{\nu, k}
$$

which we relegate to appendix F. This completes the proof of the equivalence (6.5) for arbitrary $\nu$ and $k$.

\section{Dynamical random matrix simulations with complex weights}

\subsection{Computing averages using reweighting}

In ref. [8] the analytical chRMT results for the microscopic limit of the quenched phase factor were thoroughly verified using quenched random matrix simulations. In this section we verify the unquenched analytical results using dynamical random matrix simulations. In the dynamical case the finite- $N$ corrections to the microscopic results are quite significant. Increasing the matrix size in the simulations to be close to the microscopic limit would require too much computational power, especially since dynamical simulations are already intrinsically expensive. Instead, we chose to use small-sized matrices and compare the results for the average phase factor with the finite- $N$ predictions of eq. (3.9), where the master integral is given by the sum of eqs. (4.9) and (4.17). Even then, the random matrix simulations at nonzero chemical potential are problematic since the partition function contains a complex weight. Both the real and imaginary parts of the weight function can become negative, and as a consequence the fermion determinant cannot be included in the probability distribution of a Markov Chain Monte Carlo (MCMC) simulation, i.e., we are confronted with the sign problem.

To circumvent this problem we will perform the importance sampling using an auxiliary, nonnegative, weight function, and reweight the result appropriately so that we average over the correct target ensemble. This reweighting leads to an overlap problem, when the configurations contributing most to the partition functions in both ensembles do not coincide. Note that this overlap problem always occurs in reweighting, even if the weight in the target ensemble is nonnegative. This is, for example, also an issue at zero chemical potential when using dynamical fermions with a heavier mass in the auxiliary ensemble than in the target ensemble. In the presence of a chemical potential, the overlap problem is amplified by the sign problem. Even if the auxiliary distribution has a good overlap with the target ensemble, i.e., the relevant configurations are sampled appropriately, the sign 
problem can ruin the reweighting procedure. This will happen due to large cancellations of contributions with opposite sign in the partition function of the target ensemble, which will generate large statistical errors.

Below we briefly describe the principle of reweighting. ${ }^{2}$ Using the partition function (2.3) we want to compute the unquenched expectation value

$$
\langle\mathcal{O}\rangle_{N_{f}}=\frac{1}{Z_{\nu}^{N_{f}}} \int d \varphi_{1} d \varphi_{2} w\left(\varphi_{1}\right) w\left(\varphi_{2}\right) \mathcal{D}\left[\varphi_{1}, \varphi_{2} ; \mu ;\left\{m_{f}\right\}\right] \mathcal{O}\left[\varphi_{1}, \varphi_{2}\right]
$$

with dynamical determinant

$$
\mathcal{D}\left[\varphi_{1}, \varphi_{2} ; \mu ;\left\{m_{f}\right\}\right]=\prod_{f=1}^{N_{f}} \operatorname{det}\left(D(\mu)+m_{f}\right) \equiv R e^{i \Theta} .
$$

Here, $R$ is the (nonnegative) magnitude and $e^{i \Theta}$ is the phase of $\mathcal{D}$. In the case of interest for this study, the observable will be $\mathcal{O}=e^{2 i \theta}$. To set up the reweighting formalism we introduce the weighted (or ensemble) average

$$
\langle f\rangle_{w}=\frac{\int d x w(x) f(x)}{\int d x w(x)}
$$

of $f$ with respect to $w$, with normalization $\langle 1\rangle_{w}=1$. From this definition we see that an ensemble average can be computed from an auxiliary ensemble using the reweighting relation

$$
\langle g\rangle_{w f}=\frac{\langle f g\rangle_{w}}{\langle f\rangle_{w}}
$$

This feature is useful to study ensembles with weight functions that cannot be sampled efficiently, or where the weights are not positive definite such that they cannot be used as probability distributions in importance sampling. The actual simulation constructs a Markov chain for an auxiliary ensemble, after which the expectation value of the observable in the target ensemble is computed by reweighting the observable and the partition function as given in eq. (7.4). To keep the statistical error of the reweighted observable within reasonable limits, the overlap between both ensembles should be large, i.e., the bulk of relevant configurations in both ensembles should coincide.

In this study we compare the results obtained with three different reweighting schemes [19]. The ensembles and the corresponding reweightings are as follows.

R1 Quenched simulation with full reweighting: We perform a standard quenched simulation of random matrices through direct sampling of the Gaussian weights (2.2) for the real and imaginary parts of the elements of $\varphi_{1}$ and $\varphi_{2}$, as described in appendix $E$ of ref. [8], and reweight with the dynamical fermion determinant (7.2). Using eq. (7.4) the unquenched average is computed from

$$
\langle\mathcal{O}\rangle_{N_{f}}=\frac{\left\langle R e^{i \Theta} \mathcal{O}\right\rangle_{N_{f}=0}}{\left\langle R e^{i \Theta}\right\rangle_{N_{f}=0}},
$$

\footnotetext{
${ }^{2}$ For related approaches see, e.g., [14-18].
} 
where the quenched averages in the numerator and denominator are evaluated as averages over the Monte Carlo sample.

The omission of the fermion determinant in the importance sampling will cause an overlap problem, and the reweighting factors will strongly fluctuate between configurations. These fluctuations will increase as the chemical potential grows and the sign problem becomes more tangible. In this reweighting scheme the generation of the matrices in the Markov chain is cheap, but we need a very long chain to get an acceptable accuracy.

R2 Phase quenched simulation using Metropolis with partial reweighting: In this Metropolis algorithm the matrix probability distribution consists of the product of the Gaussian weights (2.2) and the magnitude $R$ of the dynamical determinant. The measurement is then reweighted by the phase factor of the dynamical determinant,

$$
\langle\mathcal{O}\rangle_{N_{f}}=\frac{\left\langle e^{i \Theta} \mathcal{O}\right\rangle_{R}}{\left\langle e^{i \Theta}\right\rangle_{R}} .
$$

Including information about the determinant in the sampling probability should improve the overlap between the generated configurations and the significant configurations in the unquenched ensemble.

R3 Sign quenched simulation using Metropolis with minimal reweighting: As the unquenched partition function (2.3) is real, the contributions from the determinant (7.2) to the partition function only come from $R \cos \Theta$. The imaginary contributions cancel between Hermitian conjugate matrices as these have the same Gaussian probability (2.2). To minimize the variance of the reweighting factors and optimize the overlap it is therefore natural to create an auxiliary ensemble using the weight $R|\cos \Theta|$ and absorb the remaining sign of the determinant in the reweighting factor,

$$
\langle\mathcal{O}\rangle_{N_{f}}=\frac{\langle(\operatorname{sgn} \cos \Theta)(1+i \tan \Theta) \mathcal{O}\rangle_{R|\cos \Theta|}}{\langle\operatorname{sgn} \cos \Theta\rangle_{R|\cos \Theta|}},
$$

where the denominator is explicitly real because of the symmetry mentioned above. Using the absolute value of the weights as auxiliary distribution allows one to include as much information as possible about the determinant in the MCMC weights. Note that the average over the $i \tan \Theta$ term in the numerator does not vanish in this case because the observable $\mathcal{O}=e^{2 i \theta}$ also has an imaginary component. We expect this reweighting scheme to be somewhat more effective for real than for complex observables.

After performing simulations using this reweighting, we realized ${ }^{3}$ that this idea of minimal reweighting had been discussed earlier [20] in a study of the reweighting factor in lattice QCD simulations at nonzero $\mu$. In that study is was shown, based on the central limit theorem, that reweighting by $R|\cos \Theta|$ indeed minimizes the fluctuations in the reweighting factor. In ref. [20] this reweighting scheme was not

\footnotetext{
${ }^{3}$ We thank Philippe de Forcrand for bringing this earlier study to our attention.
} 
implemented since it would have been too expensive in lattice QCD. Here, we put it to a practical test on the random matrix model. (Note that scheme R3 was also rediscovered in [21].)

Note that for a complex observable one can develop more sophisticated reweighting algorithms using two Markov chains for the numerator, one for the real part of the weights and another for the imaginary part. The complication is that the chains have to be normalized properly with respect to each other, which introduces additional overhead. This will not be investigated further here.

Another partial reweighting scheme uses the $\mu$-quenched ensemble, where the matrices are sampled using a dynamical MCMC algorithm at zero chemical potential, and the contributions to the averages are reweighted by the ratio of the determinants at chemical potential $\mu$ and at $\mu=0$. For small $\mu$, this scheme is close to scheme R2, and for larger $\mu$ it is no better than scheme R1 but much more expensive. Therefore it will not be studied here.

We briefly discuss the main reweighting features of the three schemes. Clearly, the overlap problem is best handled by the sign quenched scheme, which will sample the most significant configurations of the partition function. The biggest overlap problem will be encountered by the quenched scheme, as the fermion determinant is completely ignored in the auxiliary weight function. Nevertheless, the construction of the Markov chain is very cheap in the quenched scheme, so that the overlap problem can be partially alleviated by the generation of many more configurations, which are all uncorrelated by construction. Both the sign quenched and phase quenched schemes use a Metropolis algorithm. Here the autocorrelation time has to be taken into account to select the independent configurations, which very much shortens the effective size of the Markov chain. Fortunately, the much smaller fluctuations allow us to reach a high accuracy with much fewer uncorrelated configurations than in the quenched scheme. Although the sign quenched scheme seems superior to the phase quenched one in the case of random matrix simulations, the latter can be more easily implemented in realistic theories like QCD. A phase quenched determinant for $N_{f}=2$ can be implemented by simulating a quark and a conjugate quark, whereas a sign quenched determinant does not seem to have a physical equivalent which could be implemented efficiently.

Even though the severity of the overlap problem is different for the three schemes, there is no reason why the sign problem would be improved upon in any of the schemes. Indeed, even in the sign quenched case where we sample the most significant configurations, the positive and negative contributions will balance each other more and more when the chemical potential becomes large, and the sign problem will remain. This will be confirmed in the numerical experiments discussed in the next section.

In the numerical implementation, for each matrix generated in the importance sampling we also consider its Hermitian conjugate matrix, in accordance with the symmetries of the partition function. This ensures that the sample average of the phase factor is explicitly real, which somewhat simplifies the implementation of the algorithm and the computation of the statistical error on the final result. 


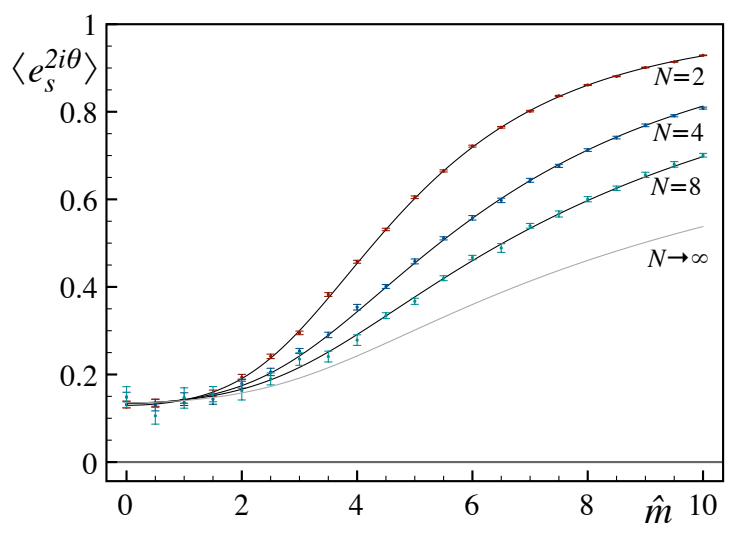

Figure 1. Average phase factor of the fermion determinant for $N_{f}=2$ as a function of the fermion mass $\hat{m}$ for $\hat{\alpha}=1.0$ and $\nu=1$ for matrix sizes $N=2,4$, and 8 . The simulation results, computed with sign quenched reweighting, are given by the data points, while the solid lines show the corresponding analytical results of eqs. (4.9) and (4.17). We also show the microscopic limit $(N \rightarrow \infty)$ of eq. (5.14).

\subsection{Numerical results}

We performed dynamical random matrix simulations at nonzero chemical potential using the three reweighting schemes described in the previous section. For each measurement we generated 1,000,000 matrices with sizes ranging from $N=2$ to 16 . In the quenched ensemble the generated matrices are uncorrelated, but for the sign and phased quenched ensembles, which are sampled using a Metropolis algorithm, successive matrices in the Markov chain are correlated. This autocorrelation effectively reduces the number of independent configurations and is taken into account appropriately when computing the statistical errors on the measurements. In addition, the statistical errors take into account the correlations between numerator and denominator in eq. (7.4). Details of the calculation of the statistical errors are given in appendix G.

In figure 1 we verify the mass dependence of the phase factor for $N=2,4$, and 8 for $N_{f}=2$ and $\nu=1$. The simulation results agree very well with the analytical predictions of eq. (3.9). Both the $\hat{m}$-dependence and the dependence on the matrix size $N$ of the matrices is reproduced. The different curves show how the large- $N$ limit is approached. For larger masses the microscopic limit is not yet reached for matrix sizes up to $N=8$, and the analytical results for finite $N$, away from the microscopic limit, are essential to explain the numerical results.

Figure 2 illustrates how the phase factor changes as a function of the chemical potential for matrix sizes $N=2,4,8$, and 16 . The results again agree very well with the analytical predictions. To probe the overlap and sign problems, which manifest themselves in the accuracy of the measurements, the simulations were performed using the three reweighting algorithms discussed above. For small chemical potential the accuracy of all three algorithms is satisfying, reflecting the fact that neither the overlap nor the sign problem is significant. However, the phase and sign quenched algorithms achieve this accuracy with much fewer independent configurations than the quenched algorithm, as a consequence of 

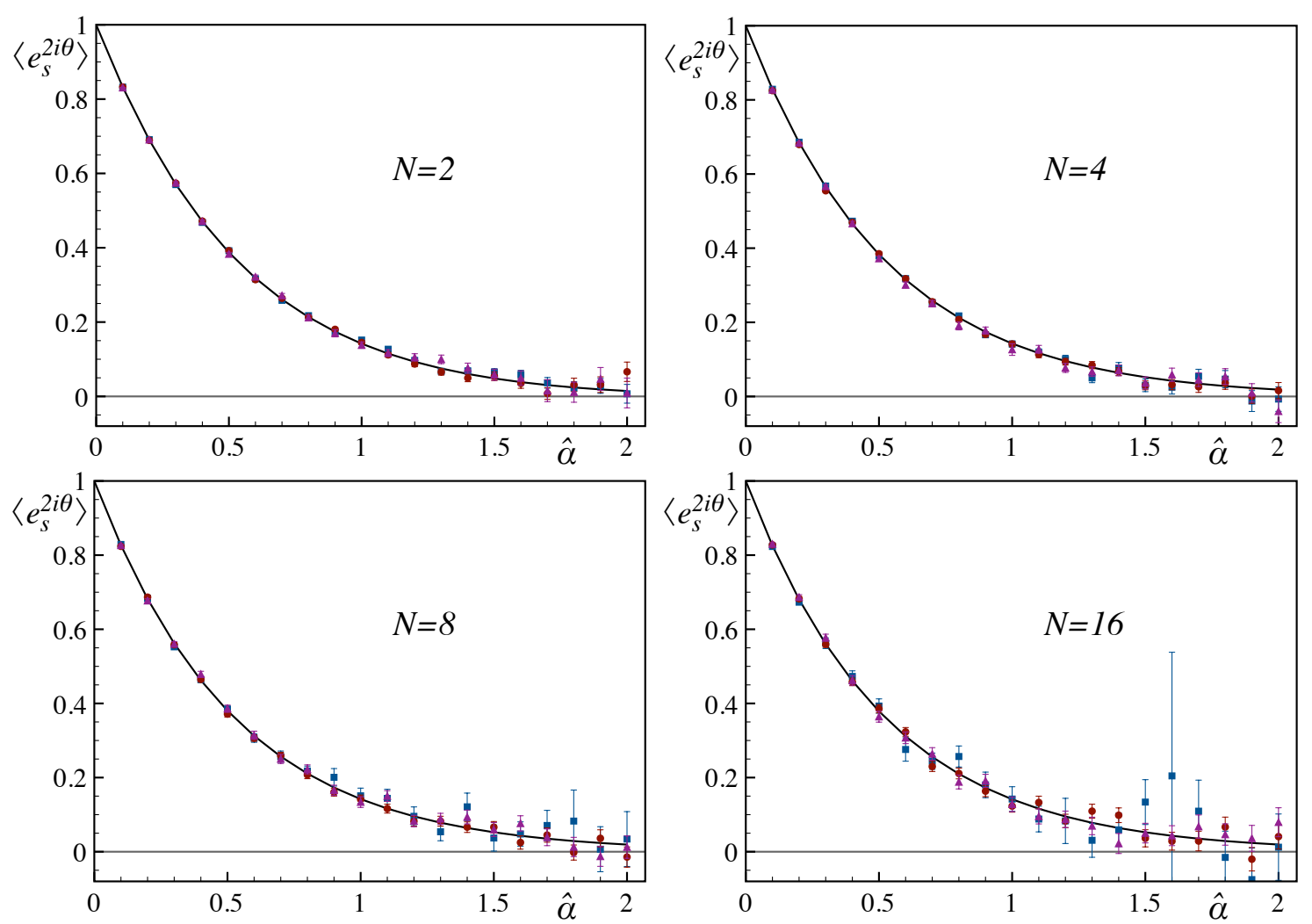

Figure 2. Average phase factor of the fermion determinant for $N_{f}=2$ as a function of the chemical potential $\hat{\alpha}$ for $\hat{m}=1.0$ and $\nu=1$ for matrix sizes $N=2,4,8$, and 16 using three different reweighting schemes. The blue squares were computed from a quenched ensemble with full reweighting, the red circles from a phase quenched simulation with partial reweighting, and the purple triangles from a sign quenched simulation with minimal reweighting.

the better overlap between target and auxiliary ensemble. The shorter trajectories would be an advantage if the measurement itself were expensive. With increasing chemical potential the error on the measurements increases as the sign problem sets in (for $\left\langle e^{2 i \theta}\right\rangle \lesssim 0.2$ ). The sign problem equally affects the different reweighting methods. As expected, the sign problem gets worse for larger $N$. In the Metropolis algorithm this deterioration for increasing $N$ is related to an increasing autocorrelation time. Even for the sign quenched algorithm, where the overlap problem is minimized, the sign problem remains as the matrices are reweighted by +1 or -1 , which leads to important cancellations and large statistical errors for large $\hat{\alpha}$.

\section{Conclusions}

Dynamical lattice simulations of QCD at nonzero baryon density are hindered by the sign problem caused by the complex fermion determinant. To investigate this problem it is helpful to employ the equivalence between the spectral properties of the Dirac operator in the $\varepsilon$-regime of QCD and chiral random matrix theory, which also holds at nonzero 
chemical potential. As the average phase factor of the fermion determinant is an important observable in the study of the sign problem, we have computed it in the framework of chiral random matrix theory.

In ref. [8] we derived an analytical formula for the average phase factor in the microscopic limit of quenched and unquenched chiral random matrix theory for general topology. In the current paper we presented an alternative derivation, leading to a different integral representation of this microscopic limit, and showed that both formulations are equivalent. In contrast to our previous work, the new formulation also gives calculable expressions for finite-sized matrices, away from the microscopic limit.

The analytical predictions of the finite- $N$ formula were verified in dynamical RMT simulations, where reweighting techniques were used to compute averages in an ensemble with a complex weight. Very good agreement was found. At large chemical potential the statistical errors grow, signifying the emergence of the sign problem.

\section{Acknowledgments}

This work was supported in part by the DFG collaborative research center SFB/TR-55. We would like to thank J. Verbaarschot and Ph. de Forcrand for useful suggestions.

\section{A Relation between orthogonal polynomials}

In this appendix we prove the identity (4.14) by induction. For $k=0$ (4.14) trivially holds for any $\nu, \ell \in \mathbb{N}$. For integer $k>0$ the recurrence relation (2.12) yields

$$
p_{\ell}^{\nu, k}(z ; \alpha)=p_{\ell+1}^{\nu, k-1}(z ; \alpha)-(\ell+\nu+k)\left(\frac{1-\alpha}{N}\right) p_{\ell}^{\nu, k-1}(z ; \alpha) .
$$

Assuming that (4.14) holds for $p_{\ell}^{\nu, k-1}$ and any $\nu, \ell \in \mathbb{N}$, we can substitute it twice in the previous equation, for $p_{\ell+1}^{\nu, k-1}$ and $p_{\ell}^{\nu, k-1}$, to find

$$
\begin{aligned}
p_{\ell}^{\nu, k}(z ; \alpha)= & \sum_{j=0}^{k-1}\left(\begin{array}{c}
k-1 \\
j
\end{array}\right) \frac{(\ell+\nu+k) !}{(\ell+\nu+k-j) !}\left(\frac{\alpha-1}{N}\right)^{j} p_{\ell+k-j}^{\nu}(z ; \alpha) \\
& +\sum_{j=0}^{k-1}\left(\begin{array}{c}
k-1 \\
j
\end{array}\right) \frac{(\ell+\nu+k) !}{(\ell+\nu+k-1-j) !}\left(\frac{\alpha-1}{N}\right)^{j+1} p_{\ell-1+k-j}^{\nu}(z ; \alpha) \\
= & p_{\ell+k}^{\nu}(z ; \alpha)+\frac{(\ell+\nu+k) !}{(\ell+\nu) !}\left(\frac{\alpha-1}{N}\right)^{k} p_{\ell}^{\nu}(z ; \alpha) \\
& +\sum_{j=1}^{k-1}\left[\left(\begin{array}{c}
k-1 \\
j
\end{array}\right)+\left(\begin{array}{c}
k-1 \\
j-1
\end{array}\right)\right] \frac{(\ell+\nu+k) !}{(\ell+\nu+k-j) !}\left(\frac{\alpha-1}{N}\right)^{j} p_{\ell+k-j}^{\nu}(z ; \alpha),
\end{aligned}
$$

where we have first shifted the index $j$ by one in the second sum and then gathered the overlapping terms in both sums. As the binomial coefficients satisfy

$$
\left(\begin{array}{c}
k-1 \\
j
\end{array}\right)+\left(\begin{array}{c}
k-1 \\
j-1
\end{array}\right)=\frac{(k-1) !}{j !(k-1-j) !}+\frac{(k-1) !}{(j-1) !(k-j) !}=\frac{k !}{j !(k-j) !}=\left(\begin{array}{l}
k \\
j
\end{array}\right)
$$


we find

$$
p_{\ell}^{\nu, k}(z ; \alpha)=\sum_{j=0}^{k}\left(\begin{array}{c}
k \\
j
\end{array}\right) \frac{(\ell+\nu+k) !}{(\ell+\nu+k-j) !}\left(\frac{\alpha-1}{N}\right)^{j} p_{\ell+k-j}^{\nu}(z ; \alpha),
$$

which shows that the identity (4.14) holds for arbitrary integer $k \geq 0$.

\section{B Chiral limit}

\section{B.1 Finite $N$}

The chiral limit of the average phase (3.9) has to be taken carefully such that the mass factors in numerator and denominator are canceled properly. For this we need to compute the limit $m \rightarrow 0$ of the Wronskian (3.11), which contains derivatives of the function $p_{\ell}^{\nu, k}$ defined in eq. (3.4). For small argument $m$ the leading-order term of this polynomial is

$$
p_{\ell}^{\nu, k}(m ; \alpha) \sim\left(\frac{1-\alpha}{N}\right)^{\ell} \frac{(\ell+\nu+k) !}{(\nu+k) !} m^{2 k},
$$

with $p$-th derivative

$$
\partial_{m}^{p} p_{\ell}^{\nu, k}(m ; \alpha) \sim\left(\frac{1-\alpha}{N}\right)^{\ell} \frac{(\ell+\nu+k) !}{(\nu+k) !} \frac{(2 k) !}{(2 k-p) !} m^{2 k-p} .
$$

Substituting these expressions in the Wronskian (3.11) and using properties of the determinant gives the leading-order result

$$
W_{n}^{\ell}\left(k_{1}, \ldots, k_{n}\right) \sim\left(\frac{1-\alpha}{N}\right)^{\ell n} m^{2 \sum_{i} k_{i}-n(n-1) / 2} \prod_{i=1}^{n} \frac{\left(\ell+\nu+k_{i}\right) !}{\left(\nu+k_{i}\right) !} 2^{n(n-1) / 2} \Delta_{n}\left(k_{1}, \ldots, k_{n}\right),
$$

where $\Delta_{n}\left(k_{1}, \ldots, k_{n}\right)$ is a Vandermonde determinant. From this we compute the chiral limit of the denominator in eq. (3.9),

$$
W_{N_{f}}^{N}\left(0,1, \ldots, N_{f}-1\right) \sim\left(\frac{1-\alpha}{N}\right)^{N N_{f}}(2 m)^{N_{f}\left(N_{f}-1\right) / 2} \prod_{i=0}^{N_{f}-1} \frac{(N+\nu+i) !}{(\nu+i) !} \prod_{\ell=1}^{N_{f}-1} \ell !,
$$

where we used the identities

$$
\Delta_{N_{f}}\left(0,1, \ldots, N_{f}-1\right)=\prod_{\ell=1}^{N_{f}-1} \ell ! \text { and } \sum_{i=0}^{N_{f}-1} i=\frac{1}{2} N_{f}\left(N_{f}-1\right) .
$$

From eq. (B.3) it is easy to see that in the limit $m \rightarrow 0$ only the Wronskian corresponding to the $k=N_{f}+1$ term in eq. (3.10) contributes to leading order, while all other terms are of higher order. The average phase (3.9) can therefore be written as

$$
\begin{aligned}
\left\langle e^{2 i \theta}\right\rangle_{m=0} & =\lim _{m \rightarrow 0} \frac{(-)^{N_{f}+1}}{(2 m)^{N_{f} N_{f}} !} \frac{W_{N_{f}+1}^{N-1}\left(0,1, \ldots, N_{f}\right)}{W_{N_{f}}^{N}\left(0,1, \ldots, N_{f}-1\right)} \mathcal{H}_{\nu, N_{f}+1}(\alpha, m) \\
& =(-)^{N_{f}+1}\left(\frac{1-\alpha}{N}\right)^{N-1-N_{f}} \frac{(N-1+\nu) !}{\left(\nu+N_{f}\right) !} \mathcal{H}_{\nu, N_{f}+1}(\alpha, m=0),
\end{aligned}
$$


where in the last step we have substituted (B.4). It now remains to take $m=0$ in $\mathcal{H}$ computed using the integrals (4.9) and (4.17). The first integral is zero as the integration range vanishes, and hence the average phase simplifies to

$$
\begin{aligned}
\left\langle e^{2 i \theta}\right\rangle_{m=0}= & {\left[\frac{(1-\alpha)^{2}}{4 \alpha}\right]^{N} \frac{\left(N+\nu+N_{f}\right) !}{(N-1) !\left(\nu+N_{f}\right) !} } \\
& \times \int_{0}^{1} d u u^{N-1}(1-u)^{N_{f}+1}\left[1+\frac{(1-\alpha)^{2} u}{4 \alpha}\right]^{-N-N_{f}-\nu-1} \\
= & {\left[\frac{(1-\alpha)^{2}}{4 \alpha}\right]^{N} \frac{\left(N_{f}+1\right) !\left(N+N_{f}+\nu\right) !}{\left(N_{f}+\nu\right) !\left(N+N_{f}+1\right) !} } \\
& \times{ }_{2} F_{1}\left(N+N_{f}+\nu+1, N ; N+N_{f}+2 ;-\frac{(1-\alpha)^{2}}{4 \alpha}\right) \\
= & \left(\frac{1-\alpha}{1+\alpha}\right)^{2 N}\left[\frac{4 \alpha}{(1+\alpha)^{2}}\right]^{\nu-1} \frac{\left(N_{f}+1\right) !\left(N+N_{f}+\nu\right) !}{\left(N_{f}+\nu\right) !\left(N+N_{f}+1\right) !} \\
& \times{ }_{2} F_{1}\left(1-\nu, N_{f}+2 ; N+N_{f}+2 ;-\frac{(1-\alpha)^{2}}{4 \alpha}\right),
\end{aligned}
$$

where in the second step we have used the integral representation of the hypergeometric function [22, eq. (15.3.1)] and in the last step we have applied the transformation ${ }_{2} F_{1}(a, b ; c ; z)=(1-z)^{c-a-b}{ }_{2} F_{1}(c-a, c-b ; c ; z)[22$, eq. (15.3.3)]. For $\nu \geq 1$ the hypergeometric function is a polynomial of degree $\nu-1$, see eq. (B.13) below, and for $\nu=1$ the result further simplifies to the $N_{f}$-independent expression

$$
\left\langle e^{2 i \theta}\right\rangle_{\nu=1, m=0}=\left(\frac{1-\alpha}{1+\alpha}\right)^{2 N}
$$

\section{B.2 Microscopic limit}

We now take the $N \rightarrow \infty$ limit of the results in appendix B.1 with $\hat{\alpha}=2 N \alpha$ fixed. In this limit we can replace $(1+\alpha)^{2}$ in the second factor and $(1-\alpha)^{2}$ in the fourth factor of (B.7) by 1 . We also have

$$
\lim _{N \rightarrow \infty}\left(\frac{1-\alpha}{1+\alpha}\right)^{2 N}=e^{-2 \hat{\alpha}}
$$

For $\nu=0$ we use the relation

$$
\begin{aligned}
\lim _{N \rightarrow \infty}{ }_{2} F_{1}\left(1, n ; N ;-\frac{N}{x}\right) & =\lim _{N \rightarrow \infty}(N-1) \int_{0}^{1} d t \frac{(1-t)^{N-2}}{(1+N t / x)^{n}}=x^{n} e^{x} \int_{x}^{\infty} d z \frac{e^{-z}}{z^{n}} \\
& =x^{n} e^{x} \Gamma(-n+1, x)
\end{aligned}
$$

where in the first step we have used the integral representation of ${ }_{2} F_{1}$ [22, eq. (15.3.1)], in the second step we have substituted $z=x+N t$ and taken the $N \rightarrow \infty$ limit, and in the third step we have used the integral representation of the incomplete gamma function $[22$, eq. (6.5.3)]. With (B.10) we obtain from (B.7)

$$
\begin{aligned}
\left\langle e_{s}^{2 i \theta}\right\rangle_{\nu=0, \hat{m}=0} & =e^{-2 \hat{\alpha}}\left[\lim _{N \rightarrow \infty} \frac{N}{2 \hat{\alpha}} \frac{N_{f}+1}{N+N_{f}+1}\right](2 \hat{\alpha})^{N_{f}+2} e^{2 \hat{\alpha}} \Gamma\left(-N_{f}-1,2 \hat{\alpha}\right) \\
& =\left(N_{f}+1\right)(2 \hat{\alpha})^{N_{f}+1} \Gamma\left(-N_{f}-1,2 \hat{\alpha}\right) .
\end{aligned}
$$


For $\nu=1$ we obtain immediately from (B.8)

$$
\left\langle e_{s}^{2 i \theta}\right\rangle_{\nu=1, \hat{m}=0}=e^{-2 \hat{\alpha}} .
$$

For $\nu \geq 2$ the first argument of the hypergeometric function in (B.7) is a negative integer, in which case we have [22, eq. (15.4.1)]

$$
{ }_{2} F_{1}(-m, b ; c ; z)=\sum_{n=0}^{m} \frac{(-m)_{n}(b)_{n}}{(c)_{n}} \frac{z^{n}}{n !}
$$

and obtain

$$
\begin{aligned}
\left\langle e_{s}^{2 i \theta}\right\rangle_{\hat{m}=0} & =e^{-2 \hat{\alpha}} \lim _{N \rightarrow \infty} \frac{\left(N_{f}+1\right) !\left(N+N_{f}+\nu\right) !}{\left(N_{f}+\nu\right) !\left(N+N_{f}+1\right) !} \sum_{n=0}^{\nu-1} \frac{(-)^{n}}{n !} \frac{(1-\nu)_{n}\left(N_{f}+2\right)_{n}}{\left(N+N_{f}+2\right)_{n}}\left(\frac{2 \hat{\alpha}}{N}\right)^{\nu-1-n} \\
& =e^{-2 \hat{\alpha}} \sum_{n=0}^{\nu-1} \frac{(\nu-1) !\left(N_{f}+n+1\right) !}{\left(N_{f}+\nu\right) ! n !(\nu-1-n) !}(2 \hat{\alpha})^{\nu-1-n} \underbrace{\lim _{N \rightarrow \infty} \frac{\left(N+N_{f}+\nu\right) !}{\left(N+N_{f}+n+1\right) ! N^{\nu-1-n}}}_{=1} \\
& =e^{-2 \hat{\alpha}} \sum_{j=0}^{\nu-1}\left(\begin{array}{c}
\nu-1 \\
j
\end{array}\right) \frac{\left(N_{f}+\nu-j\right) !}{\left(N_{f}+\nu\right) !}(2 \hat{\alpha})^{j},
\end{aligned}
$$

where in the last line we have substituted $j=\nu-1-n$.

Eqs. (B.11), (B.12) and (B.14) agree with the results previously computed in ref. [8].

\section{Proof of integral relation (6.4)}

Below we prove the integral relation (6.4). In the following, the LHS of eq. (6.4) will be denoted by $\mathcal{I}_{\nu, k}$, i.e.,

$$
\mathcal{I}_{\nu, k}=\int_{0}^{\infty} d u u^{k+1} e^{-\frac{u^{2}}{4 a}} I_{\nu}(u \sqrt{b / a}) K_{\nu+k}(u) .
$$

Using the integral representation [23, eq. (8.432.6)]

$$
K_{\nu}(u)=\frac{1}{2}\left(\frac{u}{2}\right)^{\nu} \int_{0}^{\infty} \frac{d t}{t^{\nu+1}} e^{-t-\frac{u^{2}}{4 t}} \quad \text { if } \operatorname{Re}\left(u^{2}\right)>0,
$$

which is valid for arbitrary $\nu$, we obtain

$$
\mathcal{I}_{\nu, k}=\frac{1}{2^{\nu+k+1}} \int_{0}^{\infty} \frac{d t}{t^{\nu+k+1}} e^{-t} \int_{0}^{\infty} d u u^{\nu+2 k+1} e^{-\frac{t+a}{4 a t} u^{2}} I_{\nu}(u \sqrt{b / a}) .
$$

The integral over $u$ is given in [23, eq. (6.631.1)] in terms of a confluent hypergeometric function,

$$
\begin{aligned}
\int_{0}^{\infty} & d u u^{\nu+2 k+1} e^{-\frac{t+a}{4 a t} u^{2}} I_{\nu}(u \sqrt{b / a}) \\
& =\frac{\Gamma(\nu+k+1)}{2^{\nu+1} \Gamma(\nu+1)}\left(\frac{b}{a}\right)^{\nu / 2}\left(\frac{4 a t}{t+a}\right)^{\nu+k+1}{ }_{1} F_{1}\left(\nu+k+1 ; \nu+1 ; \frac{b t}{t+a}\right) .
\end{aligned}
$$


Using the Kummer transformation [22, eq. (13.1.27)]

$$
{ }_{1} F_{1}(a ; b ; z)=e^{z}{ }_{1} F_{1}(b-a ; b ;-z)
$$

we rewrite (C.3) as

$$
\begin{aligned}
\mathcal{I}_{\nu, k} & =\frac{\Gamma(\nu+k+1)}{\Gamma(\nu+1)} 2^{k} a^{\nu+k+1}\left(\frac{b}{a}\right)^{\nu / 2} \int_{0}^{\infty} \frac{d t}{(t+a)^{\nu+k+1}} e^{-t+\frac{b t}{t+a}}{ }_{1} F_{1}\left(-k ; \nu+1 ;-\frac{b t}{t+a}\right) \\
& =\frac{\Gamma(\nu+k+1)}{\Gamma(\nu+1)} 2^{k} a\left(\frac{b}{a}\right)^{\nu / 2} e^{a+b} \int_{0}^{1} d s e^{-\frac{a}{s}-b s} s^{\nu+k-1}{ }_{1} F_{1}(-k ; \nu+1 ;-b(1-s)),
\end{aligned}
$$

where in the last step we have introduced the variable transformation $s=a /(t+a)$. Note that ${ }_{1} F_{1}(-k ; \nu+1 ; z)=k ! L_{k}^{\nu}(z) /(\nu+1)_{k}$ with the Pochhammer symbol $(a)_{n}=$ $a(a+1) \ldots(a+n-1)$ and $(a)_{0}=1$ so that the integral finally becomes

$$
\mathcal{I}_{\nu, k}=2^{k} k ! a\left(\frac{b}{a}\right)^{\nu / 2} e^{a+b} \int_{0}^{1} d s e^{-\frac{a}{s}-b s} s^{\nu+k-1} L_{k}^{\nu}(-b(1-s)) .
$$

This proves the integral relation (6.4).

\section{Proof of identity (6.8)}

In this appendix we prove the identity

$$
D_{\nu, k} \equiv \frac{d^{k}}{d s^{k}}\left[e^{-\frac{a}{s}} \frac{s^{\nu+k-1}}{(1-s)^{\nu}}\right]=e^{-\frac{a}{s}} \sum_{j=0}^{k}\left(\begin{array}{c}
k \\
j
\end{array}\right) \frac{(\nu)_{j} a^{k-j} s^{\nu-k+j-1}}{(1-s)^{\nu+j}}
$$

by induction. For $k=0$ the identity is trivially satisfied for any $\nu$, with

$$
D_{\nu, 0}=e^{-\frac{a}{s}} \frac{s^{\nu-1}}{(1-s)^{\nu}} .
$$

For $k \geq 1$ we perform one derivative in $D_{\nu, k}$ explicitly and obtain

$$
\begin{aligned}
D_{\nu, k} & =\frac{d^{k-1}}{d s^{k-1}}\left[a e^{-\frac{a}{s}} \frac{s^{\nu+k-3}}{(1-s)^{\nu}}+(\nu+k-1) e^{-\frac{a}{s}} \frac{s^{\nu+k-2}}{(1-s)^{\nu}}+\nu e^{-\frac{a}{s}} \frac{s^{\nu+k-1}}{(1-s)^{\nu+1}}\right] \\
& =\frac{d^{k-1}}{d s^{k-1}}\left[a e^{-\frac{a}{s}} \frac{s^{\nu+k-3}}{(1-s)^{\nu-1}}+(a+\nu+k-1) e^{-\frac{a}{s}} \frac{s^{\nu+k-2}}{(1-s)^{\nu}}+\nu e^{-\frac{a}{s}} \frac{s^{\nu+k-1}}{(1-s)^{\nu+1}}\right] \\
& =a D_{\nu-1, k-1}+(a+\nu+k-1) D_{\nu, k-1}+\nu D_{\nu+1, k-1} .
\end{aligned}
$$

Assuming that eq. (D.1) holds for $k-1$ we thus have

$$
\begin{aligned}
D_{\nu, k}=e^{-\frac{a}{s}} \sum_{j=0}^{k-1}\left(\begin{array}{c}
k-1 \\
j
\end{array}\right)[ & \frac{(\nu-1)_{j} a^{k-j} s^{\nu-k+j-1}}{(1-s)^{\nu+j-1}}+(a+\nu+k-1) \frac{(\nu)_{j} a^{k-j-1} s^{\nu-k+j}}{(1-s)^{\nu+j}} \\
& \left.+\frac{(\nu)_{j+1} a^{k-j-1} s^{\nu-k+j+1}}{(1-s)^{\nu+j+1}}\right]
\end{aligned}
$$




$$
\begin{aligned}
& =e^{-\frac{a}{s}}\left\{\sum_{j=0}^{k-1}\left(\begin{array}{c}
k-1 \\
j
\end{array}\right) a^{k-j}\left[\frac{(\nu-1)_{j} s^{\nu-k+j-1}}{(1-s)^{\nu+j-1}}+\frac{(\nu)_{j} s^{\nu-k+j}}{(1-s)^{\nu+j}}\right]\right. \\
& \left.+\sum_{j=0}^{k-1}\left(\begin{array}{c}
k-1 \\
j
\end{array}\right) a^{k-j-1}\left[(\nu+k-1) \frac{(\nu)_{j} s^{\nu-k+j}}{(1-s)^{\nu+j}}+\frac{(\nu)_{j+1} s^{\nu-k+j+1}}{(1-s)^{\nu+j+1}}\right]\right\} \\
& =e^{-\frac{a}{s}}\left\{\sum_{j=0}^{k-1}\left(\begin{array}{c}
k-1 \\
j
\end{array}\right) a^{k-j}\left[\frac{(\nu-1)_{j} s^{\nu-k+j-1}}{(1-s)^{\nu+j-1}}+\frac{(\nu)_{j} s^{\nu-k+j}}{(1-s)^{\nu+j}}\right]\right. \\
& \left.+\sum_{j=1}^{k}\left(\begin{array}{c}
k-1 \\
j-1
\end{array}\right) a^{k-j}\left[(\nu+k-1) \frac{(\nu)_{j-1} s^{\nu-k+j-1}}{(1-s)^{\nu+j-1}}+\frac{(\nu)_{j} s^{\nu-k+j}}{(1-s)^{\nu+j}}\right]\right\} \\
& =e^{-\frac{a}{s}}\left\{a^{k} \frac{s^{\nu-k-1}}{(1-s)^{\nu}}+\sum_{j=1}^{k-1} a^{k-j}\left[\left(\begin{array}{c}
k-1 \\
j
\end{array}\right)\left(\frac{(\nu-1)_{j} s^{\nu-k+j-1}}{(1-s)^{\nu+j-1}}+\frac{(\nu)_{j} s^{\nu-k+j}}{(1-s)^{\nu+j}}\right)\right.\right. \\
& \left.\left.+\left(\begin{array}{c}
k-1 \\
j-1
\end{array}\right)\left((\nu+k-1) \frac{(\nu)_{j-1} s^{\nu-k+j-1}}{(1-s)^{\nu+j-1}}+\frac{(\nu)_{j} s^{\nu-k+j}}{(1-s)^{\nu+j}}\right)\right]+\frac{(\nu)_{k} s^{\nu-1}}{(1-s)^{\nu+k}}\right\},
\end{aligned}
$$

where in the second step we have gathered the terms with equal powers of $a$, in the third step we have shifted the index of the second sum, and in the last step we have extracted the $j=0$ and $j=k$ terms. The binomial coefficients satisfy

$$
\left(\begin{array}{c}
k-1 \\
j-1
\end{array}\right)+\left(\begin{array}{c}
k-1 \\
j
\end{array}\right)=\left(\begin{array}{l}
k \\
j
\end{array}\right) \quad \text { and } \quad\left(\begin{array}{l}
k \\
j
\end{array}\right)=\frac{k}{j}\left(\begin{array}{c}
k-1 \\
j-1
\end{array}\right),
$$

and the Pochhammer symbols obey the identity

$$
(\nu)_{j}=(\nu-1)_{j} \frac{\nu-1+j}{\nu-1}=(\nu-1)_{j}+j(\nu)_{j-1} .
$$

Using these identities we find

$$
D_{\nu, k}=e^{-\frac{a}{s}}\left[a^{k} \frac{s^{\nu-k-1}}{(1-s)^{\nu}}+\sum_{j=1}^{k-1}\left(\begin{array}{c}
k \\
j
\end{array}\right) a^{k-j} \frac{(\nu)_{j} s^{\nu-k+j-1}}{(1-s)^{\nu+j}}+\frac{(\nu)_{k} s^{\nu-1}}{(1-s)^{\nu+k}}\right] .
$$

The first and the last term can be reabsorbed in the sum, which yields the identity (D.1).

\section{E Recurrence relation for $\mathcal{J}_{\nu, k}$}

In this appendix we show that the integral $\mathcal{J}_{\nu, k}$, defined on the LHS of eq. (6.5), satisfies the recurrence relation

$$
\mathcal{J}_{\nu, k}=-b^{\nu-1} e^{-a-b}(\nu)_{k}+\sum_{j=0}^{k}(j+1)_{k-j}\left[a b \mathcal{J}_{\nu-2, j}+(\nu-1) \mathcal{J}_{\nu-1, j}\right] .
$$

To prove this relation we substitute eq. (6.9) for $\mathcal{J}_{\nu, k}$ and rewrite the integral as

$$
\mathcal{J}_{\nu, k}=-b^{\nu-1} \int_{0}^{1} d s\left[e^{-b s}\right]^{\prime} e^{-\frac{a}{s}} \sum_{j=0}^{k}\left(\begin{array}{c}
k \\
j
\end{array}\right)(\nu)_{j} a^{k-j} s^{\nu-k+j-1}(1-s)^{k-j}
$$




$$
\begin{aligned}
= & -b^{\nu-1} e^{-a-b}(\nu)_{k}+b^{\nu-1} \int_{0}^{1} d s e^{-b s}\left[e^{-\frac{a}{s}} \sum_{j=0}^{k}\left(\begin{array}{c}
k \\
j
\end{array}\right)(\nu)_{j} a^{k-j} s^{\nu-k+j-1}(1-s)^{k-j}\right]^{\prime} \\
= & -b^{\nu-1} e^{-a-b}(\nu)_{k}+b^{\nu-1} \int_{0}^{1} d s e^{-\frac{a}{s}-b s} \sum_{j=0}^{k}\left(\begin{array}{c}
k \\
j
\end{array}\right)(\nu)_{j} a^{k-j} s^{\nu-k+j-3}(1-s)^{k-j-1} \\
& \times\left[a(1-s)+(\nu-k+j-1) s(1-s)-(k-j) s^{2}\right]
\end{aligned}
$$

where in the second step we have integrated by parts (noting that only the $j=k$ term contributes to the surface term) and in the last step we have performed the derivatives. We now manipulate the sum over $j$ in eq. (E.2), which we denote by $\mathcal{S}$. We split the coefficient $(\nu-k+j-1)$ of the second term in $\nu-1$ and $-(k-j)$ and merge this second part with the third term to obtain

$$
\mathcal{S}=\sum_{j=0}^{k}\left(\begin{array}{l}
k \\
j
\end{array}\right)(\nu)_{j} a^{k-j} s^{\nu-k+j-3}(1-s)^{k-j-1}[a(1-s)+(\nu-1) s(1-s)-(k-j) s] .
$$

Since for the third term the $j=k$ contribution to the sum vanishes we shift (for the third term only) the upper limit of the sum to $k-1$ and then $j \rightarrow j-1$, resulting in

$$
\begin{aligned}
\mathcal{S}= & \sum_{j=0}^{k}\left(\begin{array}{l}
k \\
j
\end{array}\right) a^{k-j} s^{\nu-k+j-3}(1-s)^{k-j}\left[(\nu)_{j} a+(\nu-1)_{j+1} s\right] \\
& -\sum_{j=1}^{k}\left(\begin{array}{c}
k \\
j-1
\end{array}\right)(k-j+1)(\nu)_{j-1} a^{k-j+1} s^{\nu-k+j-3}(1-s)^{k-j} \\
= & \sum_{j=0}^{k}\left(\begin{array}{c}
k \\
j
\end{array}\right) a^{k-j} s^{\nu-k+j-3}(1-s)^{k-j}\left[(\nu-1)_{j} a+(\nu-1)_{j+1} s\right],
\end{aligned}
$$

where in the last step we used the identity

$$
\begin{aligned}
\left(\begin{array}{c}
k \\
j
\end{array}\right)(\nu)_{j}-\left(\begin{array}{c}
k \\
j-1
\end{array}\right)(k-j+1)(\nu)_{j-1} & =\frac{k !(\nu)_{j}}{j !(k-j) !}-\frac{k !(\nu)_{j-1}}{(j-1) !(k-j) !} \\
& =\frac{k !(\nu)_{j-1}}{j !(k-j) !}[(\nu+j-1)-j]=\left(\begin{array}{c}
k \\
j
\end{array}\right)(\nu-1)_{j} .
\end{aligned}
$$

The Pochhammer symbols satisfy

$$
(\nu-1)_{j}=(\nu-2)_{j} \frac{\nu-2+j}{\nu-2}=(\nu-2)_{j}+j(\nu-1)_{j-1},
$$

and repeating this argument $j$ times we find

$$
(\nu-1)_{j}=\sum_{i=0}^{j}(j-i+1)_{i}(\nu-2)_{j-i} .
$$

Similarly,

$$
(\nu-1)_{j+1}=(\nu-1)_{j}(\nu-1+j)=(\nu-1)(\nu-1)_{j}+j(\nu-1)_{j},
$$


which after $j$ repetitions yields

$$
(\nu-1)_{j+1}=(\nu-1) \sum_{i=0}^{j}(j-i+1)_{i}(\nu-1)_{j-i} .
$$

Using the identity

$$
j\left(\begin{array}{l}
k \\
j
\end{array}\right)=\frac{k !}{(j-1) !(k-j) !}=k\left(\begin{array}{c}
k-1 \\
j-1
\end{array}\right)
$$

repeatedly, we find from eq. (E.7)

$$
\left(\begin{array}{c}
k \\
j
\end{array}\right)(\nu-1)_{j}=\sum_{i=0}^{j}\left(\begin{array}{l}
k \\
j
\end{array}\right)(j-i+1)_{i}(\nu-2)_{j-i}=\sum_{i=0}^{j}(k-i+1)_{i}\left(\begin{array}{c}
k-i \\
j-i
\end{array}\right)(\nu-2)_{j-i}
$$

and from eq. (E.9)

$$
\begin{aligned}
\left(\begin{array}{c}
k \\
j
\end{array}\right)(\nu-1)_{j+1} & =(\nu-1) \sum_{i=0}^{j}\left(\begin{array}{c}
k \\
j
\end{array}\right)(j-i+1)_{i}(\nu-1)_{j-i} \\
& =(\nu-1) \sum_{i=0}^{j}(k-i+1)_{i}\left(\begin{array}{c}
k-i \\
j-i
\end{array}\right)(\nu-1)_{j-i} .
\end{aligned}
$$

We now substitute the identities (E.11) and (E.12) in eq. (E.4) and find

$$
\begin{aligned}
\mathcal{S} & =\sum_{j=0}^{k} a^{k-j} s^{\nu-k+j-3}(1-s)^{k-j} \sum_{i=0}^{j}(k-i+1)_{i}\left(\begin{array}{c}
k-i \\
j-i
\end{array}\right)\left[(\nu-2)_{j-i} a+(\nu-1)(\nu-1)_{j-i} s\right] \\
& =\sum_{i=0}^{k}(k-i+1)_{i} \sum_{j=i}^{k}\left(\begin{array}{c}
k-i \\
j-i
\end{array}\right) a^{k-j} s^{\nu-k+j-3}(1-s)^{k-j}\left[(\nu-2)_{j-i} a+(\nu-1)(\nu-1)_{j-i} s\right] \\
& =\sum_{i=0}^{k}(k-i+1)_{i} \sum_{j=0}^{k-i}\left(\begin{array}{c}
k-i \\
j
\end{array}\right) a^{k-j-i} s^{\nu-k+j+i-3}(1-s)^{k-j-i}\left[(\nu-2)_{j} a+(\nu-1)(\nu-1)_{j} s\right],
\end{aligned}
$$

where in the second step we have interchanged the sums over $i$ and $j$ (see the shaded triangle in figure 3) and in the last step we have shifted the index $j$ by $i$. Looking back at the eq. (6.9) we see that $\mathcal{J}_{\nu, k}$ of eq. (E.2) can be written as

$$
\begin{aligned}
\mathcal{J}_{\nu, k} & =-b^{\nu-1} e^{-a-b}(\nu)_{k}+b^{\nu-1} \int_{0}^{1} d s e^{-\frac{a}{s}-b s} S \\
& =-b^{\nu-1} e^{-a-b}(\nu)_{k}+\sum_{i=0}^{k}(k-i+1)_{i}\left[a b \mathcal{J}_{\nu-2, k-i}+(\nu-1) \mathcal{J}_{\nu-1, k-i}\right],
\end{aligned}
$$

which, after introducing $j=k-i$, proves the relation (E.1). 


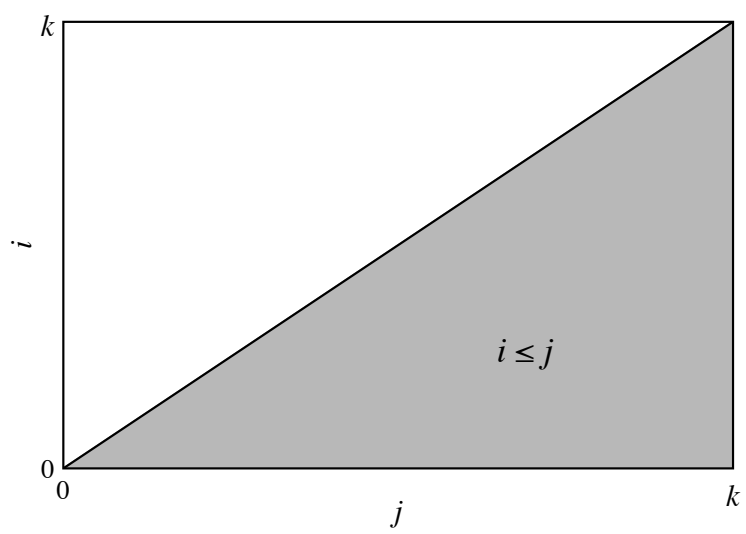

Figure 3. The range of the indices in the double sums of eq. (E.13) is given by the shaded area.

\section{F Proof of identity $(6.17)$}

With the definitions

$$
\begin{aligned}
T_{\nu, n} & =a b S_{\nu-2, n}+(\nu-1) S_{\nu-1, n} \\
\Sigma_{\nu, k} & =\sum_{n=0}^{k}(n+1)_{k-n} T_{\nu, n}
\end{aligned}
$$

we need to show that

$$
\Sigma_{\nu, k}+b^{\nu-1}(\nu)_{k}+a^{\nu-1} k !=S_{\nu, k} .
$$

We substitute the definition (6.3) of $S_{\nu, k}$ in (F.1) and perform some manipulations that are explained at the end of the string of equations,

$$
\begin{aligned}
T_{\nu, n}= & \sum_{i=0}^{\nu-3} \sum_{j=0}^{\nu-3-i} \frac{(\nu-3-i) !(\nu-3+n-j) !}{(\nu-3-i-j) ! i ! j !} b^{i+1} a^{j+1} \\
& +\sum_{i=0}^{\nu-2} \sum_{j=0}^{\nu-2-i} \frac{(\nu-1)(\nu-2-i) !(\nu-2+n-j) !}{(\nu-2-i-j) ! i ! j !} b^{i} a^{j} \\
= & \sum_{i=1}^{\nu-2} \sum_{j=0}^{\nu-2-i} \frac{(\nu-2-i) !(\nu-3+n-j) !}{(\nu-2-i-j) !(i-1) ! j !} b^{i} a^{j+1} \\
& +\sum_{i=0}^{\nu-2} \sum_{j=0}^{\nu-2-i} \frac{(\nu-1)(\nu-2-i) !(\nu-2+n-j) !}{(\nu-2-i-j) ! i ! j !} b^{i} a^{j} \\
= & \sum_{j=0}^{\nu-2} \frac{(\nu-1) !(\nu-2+n-j) !}{(\nu-2-j) ! j !} a^{j}+\sum_{i=1}^{\nu-2}\left[\sum_{j=0}^{\nu-2-i} \frac{(\nu-2-i) !(\nu-3+n-j) !}{(\nu-2-i-j) !(i-1) ! j !} b^{i} a^{j+1}\right. \\
& \left.+\sum_{j=0}^{\nu-2-i} \frac{(\nu-1)(\nu-2-i) !(\nu-2+n-j) !}{(\nu-2-i-j) ! i ! j !} b^{i} a^{j}\right]
\end{aligned}
$$




$$
\begin{aligned}
= & \sum_{j=0}^{\nu-2} \frac{(\nu-1) !(\nu-2+n-j) !}{(\nu-2-j) ! j !} a^{j}+\sum_{i=1}^{\nu-2}\left[\sum_{j=1}^{\nu-1-i} \frac{(\nu-2-i) !(\nu-2+n-j) !}{(\nu-1-i-j) !(i-1) !(j-1) !} b^{i} a^{j}\right. \\
& \left.+\sum_{j=0}^{\nu-2-i} \frac{(\nu-1)(\nu-2-i) !(\nu-2+n-j) !}{(\nu-2-i-j) ! i ! j !} b^{i} a^{j}\right] \\
= & \sum_{j=0}^{\nu-2} \frac{(\nu-1) !(\nu-2+n-j) !}{(\nu-2-j) ! j !} a^{j}+\sum_{i=1}^{\nu-2}\left[\frac{(n-1+i) !}{(i-1) !} b^{i} a^{\nu-1-i}+\frac{(\nu-1)(\nu-2+n) !}{i !} b^{i}\right. \\
& \left.+\sum_{j=1}^{\nu-2-i}(i j+(\nu-1)(\nu-1-i-j)) \frac{(\nu-2-i) !(\nu-2+n-j) !}{(\nu-1-i-j) ! i ! j !} b^{i} a^{j}\right] \\
= & \sum_{j=0}^{\nu-2}(\nu-1-j) \frac{(\nu-1) !(\nu-2+n-j) !}{(\nu-1-j) ! j !} a^{j} \\
& +\sum_{i=1}^{\nu-2} \sum_{j=0}^{\nu-1-i}(\nu-1-j) \frac{(\nu-1-i) !(\nu-2+n-j) !}{(\nu-1-i-j) ! i ! j !} b^{i} a^{j} .
\end{aligned}
$$

In the second step, we have shifted $i \rightarrow i-1$ in the first sum. In the third step, we have extracted the $i=0$ term of the second sum and merged the remaining sums. In the fourth step, we have shifted $j \rightarrow j-1$ in the first sum in square brackets. In the fifth step, we have extracted the $j=\nu-1-i$ term of the first sum and the $j=0$ term of the second sum in square brackets and merged the remaining sums. In the last step, we have written $1 /(\nu-j-2)$ ! as $(\nu-1-j) /(\nu-1-j)$ ! in the first sum. Also, we have observed that $i j+(\nu-1)(\nu-1-i-j)=(\nu-1-i)(\nu-1-j)$ and merged the three terms in square brackets into a single sum.

The result of eq. (F.4) suggests to merge the two sums since the first sum is just the $i=0$ term in the second sum, up to a missing $i=0, j=\nu-1$ term. However, such a merge needs to be done with care as the extra term is formally undefined for $n=0$. We therefore first split $\nu-1-j$ as $(\nu-1+n-j)-n$ in both sums and substitute $T_{\nu, n}$ in eq. (F.2). We switch the order of the summations and first perform the sum over $n$. Considering only the terms that depend on $n$, the sum to be evaluated is

$$
\begin{aligned}
\sum_{n=0}^{k}(n+1)_{k-n}[(\nu-1+n-j) !-n(\nu-2+n-j) !] \\
\quad=\sum_{n=0}^{k}(n+1)_{k-n}(\nu-1+n-j) !-\sum_{n=1}^{k}(n)_{k-n+1}(\nu-2+n-j) ! \\
\quad=\left[\sum_{n=0}^{k}-\sum_{n=0}^{k-1}\right](n+1)_{k-n}(\nu-1+n-j) !=(\nu-1+k-j) !
\end{aligned}
$$

where in the second step we have used $n(n+1)_{k-n}=(n)_{k-n+1}$ and observed that the $n=0$ term does not contribute to the second sum, and in the third step we have shifted $n \rightarrow n+1$ in the second sum so that only the $n=k$ term remains. Using this result we 
obtain

$$
\begin{aligned}
\Sigma_{\nu, k} & =\sum_{j=0}^{\nu-2} \frac{(\nu-1) !(\nu-1+k-j) !}{(\nu-1-j) ! j !} a^{j}+\sum_{i=1}^{\nu-2} \sum_{j=0}^{\nu-1-i} \frac{(\nu-1-i) !(\nu-1+k-j) !}{(\nu-1-i-j) ! i ! j !} b^{i} a^{j} \\
& =\sum_{i=0}^{\nu-2} \sum_{j=0}^{\nu-1-i} \frac{(\nu-1-i) !(\nu-1+k-j) !}{(\nu-1-i-j) ! i ! j !} b^{i} a^{j}-k ! a^{\nu-1} \\
& =\sum_{i=0}^{\nu-1} \sum_{j=0}^{\nu-1-i} \frac{(\nu-1-i) !(\nu-1+k-j) !}{(\nu-1-i-j) ! i ! j !} b^{i} a^{j}-k ! a^{\nu-1}-\frac{(\nu-1+k) !}{(\nu-1) !} b^{\nu-1},
\end{aligned}
$$

where in the second step we have merged the sums and corrected for the $i=0, j=\nu-1$ term, and in the last step we have extended the sum over $i$ up to $\nu-1$ and corrected for the $i=\nu-1, j=0$ term. Looking back at eq. (6.3) and using $(\nu-1+k) ! /(\nu-1) !=(\nu)_{k}$ this yields

$$
\Sigma_{\nu, k}=S_{\nu, k}-k ! a^{\nu-1}-(\nu)_{k} b^{\nu-1},
$$

which completes the proof.

\section{G Error estimation for the random matrix simulations}

When using reweighting the ensemble average (7.4) can be written as $\bar{z}=\bar{x} / \bar{y}$, and the error on $\bar{z}$ is given by the usual error propagation formula

$$
\sigma_{\bar{z}}=|\bar{z}| \sqrt{\frac{\sigma_{\bar{x}}^{2}}{\bar{x}^{2}}+\frac{\sigma_{\bar{y}}^{2}}{\bar{y}^{2}}-2 \rho_{x y} \frac{\sigma_{\bar{x}}}{\bar{x}} \frac{\sigma_{\bar{y}}}{\bar{y}}},
$$

where $\sigma_{\bar{x}}=\sigma_{x} / \sqrt{N}$ and $\sigma_{\bar{y}}=\sigma_{y} / \sqrt{N}$ are the standard errors of $\bar{x}$ and $\bar{y}, \sigma_{x}$ and $\sigma_{y}$ are the square roots of the sample variances of $x$ and $y, \rho_{x y}$ is the correlation coefficient of $x$ and $y$, and $N$ is the sample size.

In the quenched simulation all configurations are independent and the standard errors are computed using the total sample size $N$. For the phase-quenched and sign-quenched simulations the ensembles are generated using a Metropolis algorithm, and the autocorrelations in the Markov chain have to be taken into account by modifying the standard errors to

$$
\sigma_{\bar{x}}=\sqrt{\frac{2 \tau_{\text {int }, x}}{N}} \sigma_{x}, \quad \sigma_{\bar{y}}=\sqrt{\frac{2 \tau_{\text {int }, y}}{N}} \sigma_{y},
$$

where $\tau_{\text {int }, x}$ and $\tau_{\text {int }, y}$ are the integrated autocorrelation times for $x$ and $y$ [24]. In eq. (G.1) the correlation coefficient $\rho_{x y}$ is computed over the complete sample, and it is assumed that $\rho_{x y}$ is not affected by different autocorrelation times for $x$ and $y$.

\section{References}

[1] P. de Forcrand, Simulating QCD at finite density, PoS LAT2009 (2009) 010 [arXiv: 1005.0539]. 
[2] J. Gasser and H. Leutwyler, Thermodynamics of chiral symmetry, Phys. Lett. B188 (1987) 477.

[3] E. V. Shuryak and J. J. M. Verbaarschot, Random matrix theory and spectral sum rules for the Dirac operator in QCD, Nucl. Phys. A560 (1993) 306 [hep-th/9212088].

[4] J. J. M. Verbaarschot and T. Wettig, Random matrix theory and chiral symmetry in QCD, Ann. Rev. Nucl. Part. Sci. 50 (2000) 343 [hep-ph/0003017].

[5] F. Basile and G. Akemann, Equivalence of $Q C D$ in the epsilon-regime and chiral random matrix theory with or without chemical potential, JHEP 12 (2007) 043 [arXiv:0710.0376].

[6] K. Splittorff and J. J. M. Verbaarschot, The QCD sign problem for small chemical potential, Phys. Rev. D75 (2007) 116003 [hep-lat/0702011].

[7] J. Han and M. A. Stephanov, Random matrix study of the QCD sign problem, Phys. Rev. D78 (2008) 054507 [arXiv:0805.1939].

[8] J. Bloch and T. Wettig, Random matrix analysis of the QCD sign problem for general topology, JHEP 03 (2009) 100 [arXiv:0812.0324].

[9] J. C. Osborn, K. Splittorff, and J. J. M. Verbaarschot, Chiral condensate at nonzero chemical potential in the microscopic limit of QCD, Phys. Rev. D78 (2008) 065029 [arXiv: 0805.1303].

[10] J. C. Osborn, Universal results from an alternate random matrix model for QCD with a baryon chemical potential, Phys. Rev. Lett. 93 (2004) 222001 [hep-th/0403131].

[11] G. Akemann, J. C. Osborn, K. Splittorff, and J. J. M. Verbaarschot, Unquenched QCD Dirac operator spectra at nonzero baryon chemical potential, Nucl. Phys. B712 (2005) 287 [hep-th/0411030].

[12] M. C. Bergere, Biorthogonal polynomials for potentials of two variables and external sources at the denominator, hep-th/0404126.

[13] G. Akemann and A. Pottier, Ratios of characteristic polynomials in complex matrix models, J. Phys. A37 (2004) L453 [math-ph/0404068].

[14] K. N. Anagnostopoulos and J. Nishimura, New approach to the complex-action problem and its application to a nonperturbative study of superstring theory, Phys. Rev. D66 (2002) 106008 [hep-th/0108041].

[15] J. Ambjørn, K. N. Anagnostopoulos, J. Nishimura, and J. J. M. Verbaarschot, The factorization method for systems with a complex action - A test in random matrix theory for finite density QCD, JHEP 10 (2002) 062 [hep-lat/0208025].

[16] Z. Fodor, S. D. Katz, and C. Schmidt, The density of states method at non-zero chemical potential, JHEP 03 (2007) 121 [hep-lat/0701022].

[17] S. Ejiri, On the existence of the critical point in finite density lattice QCD, Phys. Rev. D77 (2008) 014508 [arXiv: 0706.3549].

[18] K. N. Anagnostopoulos, T. Azuma, and J. Nishimura, General approach to the sign problem: Factorization method with multiple observables, Phys. Rev. D83 (2011) 054504 [arXiv: 1009.4504].

[19] J. C. R. Bloch and T. Wettig, Random matrix analysis of the QCD sign problem, PoS LAT2009 (2009) 186 [arXiv:0910.1206]. 
[20] P. de Forcrand, S. Kim, and T. Takaishi, QCD simulations at small chemical potential, Nucl. Phys. Proc. Suppl. 119 (2003) 541 [hep-lat/0209126].

[21] S. D. H. Hsu and D. Reeb, On the sign problem in dense QCD, Int. J. Mod. Phys. A25 (2010) 53 [arXiv:0808.2987].

[22] M. Abramowitz and I. A. Stegun, Handbook of Mathematical Functions with Formulas, Graphs, and Mathematical Tables. Dover, New York, 9th ed., 1973.

[23] I. Gradshteyn and I. Ryzhik, Table of Integrals, Series and Products. Academic Press, San Diego, 5th ed., 1994. [errata: www.mathtable.com/errata/gr6_errata.pdf].

[24] N. Madras and A. D. Sokal, The Pivot algorithm: A highly efficient Monte Carlo method for the self-avoiding walk, J. Statist. Phys. 50 (1988) 109. 